\title{
Influencia de la infraestructura bibliotecaria en el desarrollo de producción científica en universidades mexicanas inmersas en procesos de acreditación
}

\author{
Laura Patricia MURGuía JÁQUEZ \\ pmurguia@uach.mx \\ Javier TARANGO ORTIZ \\ tj.88888@hotmail.com \\ José Refugio Romo GonZÁLEZ \\ jromo@uach.mx \\ Gerardo ASCENCIO BACA \\ gbaca@uach.mx \\ Universidad Autónoma de Chihuahua. Facultad de Filosofía y Letras
}

Recibido: Junio 2013

Aceptado: Agosto 2013

Resumen: El papel que juegan las bibliotecas universitarias en los procesos educativos ha sido considerado relevante, debido a sus contribuciones de apoyo a las actividades académicas y científicas que suceden en los centros de educación superior, tanto con fines de transferencia como de generación de conocimiento por parte de diversos actores que interactúan en relación con los recursos informativos. Este artículo, a partir de la integración de un modelo teórico simplificado, analiza el comportamiento que observan docentes e investigadores de 33 universidades mexicanas que han experimentado algún proceso de acreditación, en relación con diversos indicadores de producción científica (evaluada en tres aspectos generales: productividad, calidad e innovación), al tiempo que se estudia a la infraestructura bibliotecaria disponible, la cual comprende: volúmenes impresos disponibles, suscripciones a revistas impresas, suscripciones a bases de datos, cantidad de usuarios docentes, cantidad de usuarios estudiantes y superficie física en metros cuadrados. A partir del análisis estadístico y la ponderación de datos, debido a la disparidad en el tamaño de las universidades participantes, se desarrollaron una serie de correlaciones, mismas que propiciaron la identificación de aquellos indicadores de infraestructura física que han tenido influencia en los de producción científica, infiriéndose además, patrones de uso y acceso a los recursos informativos por parte de los docentes e investigadores.

Palabras Clave: Producción científica; Comunicación de la ciencia; Infraestructura bibliotecaria; Universidades mexicanas; bibliotecas académicas acreditadas. 


\title{
Influence of library infrastructure in developing scientific production in teachers and researchers from Mexican universities
}

\begin{abstract}
The role of university libraries in education has been considered relevant, because this to their contributions to support the academic and scientific activities that occur in higher education institutions, both for knowledge transfer and generation, by various actors interacting in relation to information resources. This article, based on the integration of a simplified theoretical model analyzes the behavior they see teachers and researchers from 33 universities in Mexico participating in accreditation processes, on various scientific production indicators (evaluated in three broad areas: quality, production and innovation), the while studying at the library infrastructure available, such as: printed volumes available, subscriptions to print journals, subscriptions to databases, quantity of teachers as users, quantity of students as users and physical area in square meters. From the statistical analysis and data weighting, due to the disparity in the size of the participating universities, developed a series of correlations; same that led to the identification of those physical infrastructure indicators have influenced those of scientific production, inferring addition, patterns of use and access to information resources by teachers and researchers.
\end{abstract}

Keywords: Scientific production: Communication of science; Library infrastructure; Mexican universities; accredited academic libraries.

\section{INTRODUCCIÓN}

Ante las exigencias de una sociedad cambiante basada en conocimiento, las universidades actuales enfrentan el reto constante de adaptación a nuevas condiciones de trabajo, las cuales han sobrepasado en mucho, la alta concentración en actividades relacionadas meramente con la transferencia de conocimiento, fundamentadas a través de acciones de docencia o de búsqueda y adquisición de conocimiento, generalmente por medio de procesos de acceso a información basada en documentos.

La identificación de los actores centrales que forman las comunidades académicas y científicas, en un tiempo enfocadas en los estudiantes como eje principal, ahora se revierten en los docentes e investigadores, como piezas clave para integrar a su abanico de actividades cotidianas, aquellas que superan en mucho la transferencia de conocimiento, sino cada vez más apuntaladas hacia acciones relacionadas con la gestión, la investigación y la comunicación de la ciencia a partir de participar en publicaciones de calidad, soportadas por grados académicos preferentes (doctorado) y distinciones de reconocimiento basadas en niveles de producción académica y científica.

Ante la definición del docente-investigador como elemento fundamental del contexto en que se basa el presente estudio, cabe mencionar que las universidades actuales mantienen como reto el desarrollo de su personal docente considerando diversos factores, tales como: el aumento de su formación superior a través de la adquisición de grados académicos (para el caso de profesores contratados con 
antelación); el acercamiento de profesores con formación académica suficiente y con producción científica de calidad que garantice su continuidad de trabajo; los procesos de internacionalización de la producción científica a través de ampliar los radios de divulgación del conocimientos, circunscribiéndose menos a entornos locales; los procesos de colaboración académica entre grupos de docentes e investigadores afines en intereses científicos, incluso personales, que propicien a su vez la reorganización de conocimientos en cuanto a especialización en líneas de generación y aplicación de conocimiento. Esto por mencionar las preocupaciones principales en relación con los cuerpos docentes e investigativos, proyectando su consecuencia en la medición de la eficiencia de los centros educativos.

En relación con los procesos de producción científica, las universidades actuales demandan la generación de nuevos espacios o el fortalecimiento de los existentes que permitan la generación de conocimiento. En tales espacios se contemplan laboratorios, centros de investigación, medios de divulgación, pero especialmente bibliotecas universitarias capaces de cubrir, con su infraestructura física y de información, las necesidades que demandan los académicos para desarrollar su trabajo investigativo.

Los países de economías emergentes, como es el caso de México, mantienen la intención de adherirse a los cambios que la sociedad del conocimiento demanda, las universidades no son la excepción. Las organizaciones educativas deben responder a las nuevas expectativas sociales en relación con la educación, la definición de competencias académicas en sus egresados y la enseñanza científica.

Este último aspecto es el que toma mayor relevancia en la determinación de los propósitos del presente artículo, ya que es considerado un medio preciso para identificar la calidad de las universidades a partir de sus niveles de generación de conocimiento. En este sentido, las bibliotecas universitarias deberán también adecuarse a la actividad científica universitaria, una vez que integren fondos documentales enfocados a líneas de investigación que se cultiven en los centros educativos; el docente e investigador inicia una movilidad constante, no sólo desde la perspectiva física, sino desde sus acciones medidas en la consulta de información de distintas latitudes y la divulgación de conocimiento en radios cada vez más amplios, los cuales en sí, tienden a ser más competitivos.

\section{BIBLIOTECAS UNIVERSITARIAS Y PRODUCCIÓN CIENTÍFICA}

Considerar a la biblioteca universitaria dentro de los ámbitos educativos y establecer su influencia en los procesos de producción científica o en cualquier actividad que contribuya en los procesos educativos es una tarea compleja, incluso difícil de probar, sin embargo, ya ha sido previamente estudiada por distintos autores, al menos al nivel de preocupación sobre el rol de la información 
sistematizada en una biblioteca y las formas como contribuye en los procesos de generación de investigación y conocimiento (Tarango y Hernández-Orozco, 2010).

Desde hace tres décadas, Roe (1981) mostró su preocupación sobre el rol de las bibliotecas universitarias y su proyección en las instituciones educativas en las que están inmersas, las formas como se debió observar su evolución y la necesidad de generar nuevas formas de involucramiento de los usuarios, en este caso, que los docentes e investigadores estén al nivel de análisis de comportamiento de los estudiantes en cuanto a frecuencia y repercusiones por el uso de los recursos informativos.

Aunque no hace una diferenciación entre los tipos de usuarios comunes a las bibliotecas universitarias, Lau (2001) consideró la relación de los ámbitos educativos y de investigación con el uso de los recursos de información, además de manifestar la necesidad de un espacio que funcione como laboratorio de ideas, sustentado en repertorios de calidad y qué sirva como un enlace a las redes mundiales de datos. Agrega también que tal escenario, tendrá entre otras funciones, la contribución al desarrollo de cuadros profesionales de calidad acordes a las demandas de la sociedad, capaces de crear cambios científicos, tecnológicos y laborales.

Ante la inminente necesidad de centrar la atención en el docente como usuario de la biblioteca universitaria, Bustos González (2007), sugiere de manera puntual que los académicos e investigadores participen activamente en la gestión de aprendizaje y conocimiento, como una parte fundamental en su actualización permanente, ya que los recursos de información disponibles pueden contribuir al aumento de la producción científica proyectada en publicaciones.

$\mathrm{La}$ biblioteca universitaria, al pretender un papel protagónico en las contribuciones en la producción científica que se generan en las universidades, de una forma indirecta de formación de recursos humanos hacia la investigación (Wilburn, 2004; Lopera, 2008). En ese mismo sentido, las tentativas de reforma educativa hacia la producción científica, que sólo se manifiestan en una línea discursiva, no tendrán ningún impacto si no consideran en su conjunto los diversos factores que influyen en la generación de nuevos conocimientos.

Es imprescindible dejar de ver a la biblioteca universitaria como un simple conjunto de recursos, que aunque posteriormente se ponderan como fundamentales para definir condiciones de infraestructura bibliotecaria, y considerarla como una plataforma capaz de propiciar la generación de conocimiento, cuya proyección se manifieste en activos intelectuales regularmente asociados con publicaciones científicas, propiciando nuevo poder y valor a la competencia de los académicos a partir de su capacidad de generación de conocimiento (Cabral Vargas, 2007).

Se espera entonces, que la biblioteca universitaria cumpla dos funciones principales en relación con la producción científica: la primera, basada en su infraestructura es capaz de reunir, procesar, difundir, almacenar y propiciar el uso de la información; la segunda, buscar mecanismos que permitan la generación de nuevo conocimiento de valor, estableciendo ciertos parámetros de calidad como: 
autoridad, audiencia, actualidad, cobertura, precisión, accesibilidad objetividad y costo (Fushmini, Mallo y Pichinini, 2005). La influencia de la biblioteca universitaria deberá estar relacionada con la difusión del conocimiento generado en las propias instituciones de educación superior, por lo tanto, se convierte en memoria académica y científica de las organizaciones educativas, reflejadas en producción académica y científica, entendida como el conjunto de trabajos editados generada por docentes e investigadores a través de artículos, libros, capítulos de libros, tesis y proyectos de investigación.

La inclusión de docentes e investigadores a los procesos permanentes de generación de conocimiento, requiere de un aliado debidamente organizado. En este sentido es necesario considerar que su operatividad depende de: tecnología avanzada y personal bibliotecario altamente capacitado en actividades de aprendizaje individual y colaborativo (Bustamante Rodríguez y Padial León, 2003; Webb y Grimwood-Jones, 2003); tener bibliotecas físicas y digitales actualizadas que respalden los programas académicos y proyectos de investigación; y programas académicos formales e informales orientados a la administración de información y la ingeniería del conocimiento (Cabral Vargas, 2007).

La conservación de la memoria académico-científica de las universidades recae en la biblioteca universitaria, de tal forma que se convierta en una fuente confiable -a través de sus indicadores cualitativos y cuantitativos de producción científica, que facilite la gestión, el acceso y la evaluación informativa. El funcionamiento efectivo de la biblioteca universitaria como un resguardo de documentos y de proporción de servicios de información requieren de un orden adecuado y de una infraestructura suficiente, en donde convergen los conceptos de informaciónproducción científica (Lara Orduño, 2004)

Para efectos de la calidad de las bibliotecas universitarias, su infraestructura es determinante, especialmente cuando es entendida como todos aquellos elementos que ayudan a satisfacer las necesidades de información de los usuarios. En este tipo de infraestructura sobresalen las colecciones de libros, revistas y bases de datos, todas ellas, tanto físicas como en formato electrónico. También se considera el espacio físico como un elemento que apoya a los usuarios para alcanzar sus objetivos de enseñanza-aprendizaje, así como la producción de conocimiento.

De este modo, un espacio bibliotecario idóneo para el beneficio de la comunidad generadora de producción académica y científica, estimulará y dará confianza al investigador de cualquier área. Dependiendo de forma específica de la disciplina de investigación, los escenarios para la generación de conocimiento pueden ser diversos (como laboratorios), sin embargo en los que todos coinciden es en la bibliotecas universitarias.

La relación fundamental entre las bibliotecas universitarias (considerando sus servicios e infraestructura) y la producción científica, sucederá cuando los académicos e investigadores como usuarios, sean un vínculo posterior con la publicación de los resultados obtenidos usando diversas fuentes de información 
que se relacionan con su campo de conocimiento y con fines de divulgación. Se espera entonces, que para que la producción científica crezca y sea reconocible por su calidad, es de suma importancia que se cuente con infraestructura bibliotecaria acorde a las necesidades de los usuarios.

Gómez (2002), explica que "si la biblioteca existe para cumplir una serie de requerimientos educativos, informativos, culturales, recreativos, de investigación, etc., se hace necesario la existencia de edificios bibliotecarios que permitan esas funciones con una utilización funcional, segura, económica y a poder ser, confortable" (p. 6). La suficiencia de los espacios físicos, adecuados y flexibles, en la infraestructura bibliotecaria, genera seguridad y permite un mejor desempeño en los recursos disponibles, los espacios serán mejor aprovechados, especialmente si se cuentan con recursos electrónicos (Breeding, 2011).

La cuestión de la infraestructura bibliotecaria no es al azar, debe estar vinculada con los requerimientos de producción de conocimiento, además de que debe contar con ciertos estándares que permitan su evaluación y definición de la forma como se desarrollará en un futuro.

En el caso de México, las diferentes normas y directrices que rigen a las bibliotecas universitarias sólo se consideran como un modelo a seguir, ya que cada una se desarrolla de acuerdo a diferentes contextos. Una de las propuestas concretas en relación con la contribución de la infraestructura bibliotecaria con la producción científica son las que establece el Consejo Nacional para Asuntos Bibliotecarios de las Instituciones de Educación Superior (CONPAB-IES, 2005), reforzado por el análisis hecho por López Ruelas y Cortés (2011), que pudiera ser lo más aproximado a México en cuestiones de:

a) Regulaciones bibliotecarias en relación con los espacios necesarios por docente e investigador (de 3 a 4.5 metros cuadrados de acuerdo a los niveles académicos).

b) La definición de número de espacios por usuario alumno inscrito y por docente de tiempo completo.

c) El número de volúmenes y títulos disponibles por tipo de material (indica una relación con alumnos usuarios, docentes usuarios y por programas académicos).

Por lo anterior, las Normas para bibliotecas de Instituciones de Educación Superior e Investigación de CONPAB-IES (Consejo Nacional para Asuntos Bibliotecarios de las Instituciones de Educación Superior), refieren la necesidad de implementar lineamientos y directrices generales para la operación y desarrollo de las bibliotecas académicas mexicanas.

En países como en Estados Unidos la normatividad de infraestructura bibliotecaria viene de tiempo (Bryant, 1989), en donde desde entonces, se da importancia a los espacios bibliotecarios en cualquier tipo de centro de información. 
Específicamente para las bibliotecas universitarias la Association of College \& Research Libraries, ACRL (1994) definió parámetros de espacios para albergar colecciones, para el estudio y la investigación, así como para los procesos asociados y tareas de servicio al público, sin olivar la provisión de espacios para los servicios automatizados en un entorno debidamente controlado. Por lo anterior se asume que la biblioteca debe contar con el espacio suficiente para albergar las colecciones, para el estudio y la investigación, así como para la prestación de servicios.

Las bibliotecas mexicanas, por su condición presupuestaria principalmente y de espacios, no cuentan con los suficientes materiales que se producen en las diferentes áreas de conocimiento, por lo que una medida que se ha tomado siguiendo patrones de países desarrollados, es la de implementar la cooperación entre bibliotecas del mismo sistema bibliotecario e incluso con otras instituciones para suplir las carencias y facilitar el uso de materiales para la investigación.

\section{DISEÑO DE LA INVESTIGACIÓN}

A continuación se incluyen los elementos principales que definen la estructura fundamental del estudio que especifica la naturaleza global de la intervención:

\subsection{PLANTEAMIENTO Y JUSTIFICACIÓN DEL PROBLEMA}

Los estudios realizados en relación con la evaluación bibliotecaria han tomado como medida la determinación del tamaño de sus colecciones físicas y electrónicas, así como el comportamiento específico de sus usuarios limitados a la frecuencia de uso. Sin embargo, se considera que las contribuciones de las bibliotecas universitarias deberán ser medidas en relación a su influencia en la educación y en la producción científica desarrollada por académicos, tanto de forma directa, así como considerando la influencia que puedan tener en estudiantes a través de investigaciones, tesis y proyectos.

Las aplicaciones en el uso de la información deberá estar proyectada hacia la generación de conocimiento y por lo tanto, a la solución de problemas concretos. Pocos estudios identificados intentan comprobar que a mayor infraestructura bibliotecaria mayor producción científica, ya que la limitante se manifiesta cuando no es posible garantizar que los recursos de infraestructura bibliotecaria disponible en las universidades, representen un reflejo de la actividad en su uso por docentes e investigadores o que finalmente sean dependientes de recursos externos para el logro de sus objetivos investigativos y de divulgación del conocimiento.

Existe la necesidad de que las bibliotecas universitarias desempeñen un rol activo de soporte a la actividad investigadora de las instituciones de educación superior y concretamente a la difusión de la producción científica, teniendo en cuenta que es un factor estratégico decisivo en el posicionamiento de las instituciones académicas, 
en la obtención de recursos económicos y en la evaluación de los investigadores (Blanco y Casaldáliga, 2012). Por lo anterior, puede verse que la investigación siempre estará ligada no solo al aprendizaje, sino al uso de recursos económicos implicados con las colecciones, los espacios, bases de datos y aplicación de la tecnología. Además, estos servicios se vinculan a los procesos de evaluación de los investigadores que está muy ligada a la producción académica.

En la actualidad las bibliotecas académicas en general también guardan relación con los procesos educativos modernos. Para ello, es necesario hacer un uso eficiente de todos los recursos con los que se cuenta para permitir a los usuarios y docentes potencializarlos en la producción de documentos científicos. Los cuales, posteriormente se difunden a través de publicaciones de calidad. Todos los recursos destinados a generar productos científicos y académicos se volverán eficientes con el apoyo del uso de la biblioteca universitaria. De ahí que a mayor calidad de realización investigativa basada en la biblioteca universitaria se obtendrán más posibilidades de proyectos para alcanzar publicaciones de alto nivel.

\subsection{OBJETIVO}

El objetivo del presente estudio es establecer la relación que existe entre la producción científica gestada por académicos e investigadores de universidades mexicanas y la infraestructura bibliotecaria disponible. Por tanto, de forma más explícita, el estudio propone: Desarrollar un análisis comparativo entre los niveles de productividad, calidad e innovación científica de las universidades mexicanas que pertenecen al CONPAB-IES y la disposición de recursos de infraestructura bibliotecaria desde la perspectiva de sus usuarios, disponibilidad de recursos informativos (físicos y electrónicos) y espacios físicos.

De acuerdo a lo anterior y considerando a la producción científica como uno de los indicadores para medir la calidad de las universidades, se pretende exponer el grado de importancia de los insumos involucrados en el proceso de investigación y a partir de éstos, delimitar la significancia que tienen cada uno de los elementos de la infraestructura bibliotecaria. Por tanto, se analiza:

a) La producción científica como medio para establecer diversos campos de edición de documentos de calidad, publicados para su divulgación.

b) Los objetivos de la buena producción científica tienen amplia relación con los niveles de formación académica de los docentes e investigadores que la desarrollan, así como de los programas académicos en que están inmersos.

c) La validez en la calidad de la producción científica, justifica el quehacer de la biblioteca como entidad de soporte académica. 


\subsection{DELIMITACIÓN DEL ESTUDIO}

Para el desarrollo de la investigación, fueron elegidas las universidades que integran el CONPAB-IES, constituida por 38 entidades de educación superior, de las cuales se logró recolectar la información relacionada con la infraestructura bibliotecaria a un total de 33, debido a que cinco no respondieron a la solicitud de datos (anexo 1).

De las 33 universidades que proporcionaron datos sobre infraestructura bibliotecaria (correspondientes al 2011 integrados en 2012), se logró obtener la información, correspondiente al mismo período, relacionada con la producción científica según los parámetros del modelo teórico simplificado generado para los propósitos del presente estudio y presentado posteriormente. Para lograr comparar variables, existió la necesidad de que hubiera concordancia en la disposición de datos, tanto de la infraestructura bibliotecaria como de producción científica.

Tomando en consideración la clasificación de las universidades mexicanas hecha por Galaz Fontes (1998), quien especifica no existir en México una identificación clara sobre el tamaño y características de las universidades, se identifican: seis universidades grandes (con más de 40 mil estudiantes), 13 medianas (15 a 39 mil estudiantes) y 14 chicas (menos de 15 mil estudiantes). De todas ellas una es privada, 29 públicas estatales y 3 públicas federales.

La elección de las universidades del CONPAB-IES (2013) como población de esta investigación, es debido a que es una organización que promueve el desarrollo de servicios bibliotecarios en instituciones académicas y de investigación en México, tomando como base una normatividad, todo con el fin de unificar, sin necesidad de uniformar, el criterio en asuntos concernientes al ejercicio bibliotecológico y al desarrollo de los servicios profesionales.

\subsection{METODOLOGÍA}

Este trabajo es una investigación de tipo descriptivo y correlacional, en el cual se busca identificar propiedades, características y perfiles importantes del comportamiento en la producción de 33 universidades pertenecientes a CONPABIES, cuyas variables de análisis, tanto dependientes e independientes, posteriormente son correlacionadas. Las actividades principales desarrolladas fueron:

a) Identificación de las universidades que conforman a la organización CONPAB-IES, considerando para ello, datos específicos, tales como: nombre de la universidad, datos del responsable del proyecto de bibliotecas y formas de localización, esto con el fin de establecer comunicación directa, vía telefónica o usando correo electrónico, y recabar los siguientes datos que corresponden a las variables relacionadas con la infraestructura bibliotecaria: cantidad de volúmenes impresos, número de suscripciones a revistas impresas, número de suscripciones a bases de datos, usuarios docentes, usuarios estudiantes y 
superficie física en metros cuadrados, correspondientes al ejercicio 2011 e integrados en el 2012.

b) Una vez recabados los datos considerados en el punto anterior, se procedió a identificar los correspondientes a producción científica de aquellas universidades en las que fue posible obtener la información completa relacionada con su infraestructura bibliotecaria. Se accedió al observatorio científico desarrollado por la Universidad Nacional Autónoma de México (UNAM, 2013), llamado Estudio Comparativo de Universidades Mexicanas (ECUM), para recolectar del año 2011, integrados en 2012, la información correspondiente a los indicadores siguientes: datos institucionales (personal docente y matricula); indicadores de investigación (personal docente miembro del Sistema Nacional de Investigadores - SNI); indicadores de revistas académicas (revistas del directorio y catálogo Latindex y revistas incluidas en el índice de Revistas Mexicanas de Investigación Científica y Tecnológica del Consejo Nacional de Ciencia y Tecnología - CONACYT); y, indicadores de divulgación de la ciencia comprendiendo número de revistas generadas por cada universidad y número de artículos publicados en el Institute for Scientific Information (ISI), SCOPUS, PERDIÓDICA y CLASE (todas las entidades que son explicadas posteriormente).

c) Se identificó un modelo teórico simplificado (figura 1), el cual se constituye de dos área fundamentales: la evaluación de la producción científico-tecnológica, derivada del concepto sobre competitividad organizacional planteado por Palacios Banco y Vergas Chanes (2009), quien afirma que la competitividad de toda organización se integra y sustenta en tres elementos: productividad, calidad e innovación; y la infraestructura bibliotecológica, basada en seis indicadores: volúmenes impresos, suscripciones a revistas impresas, suscripciones a bases de datos, usuarios docentes, usuarios estudiantes y dimensiones físicas. Las variables independientes son: matrícula de estudiantes y docentes.

Figura 1. Modelo teórico simplificado
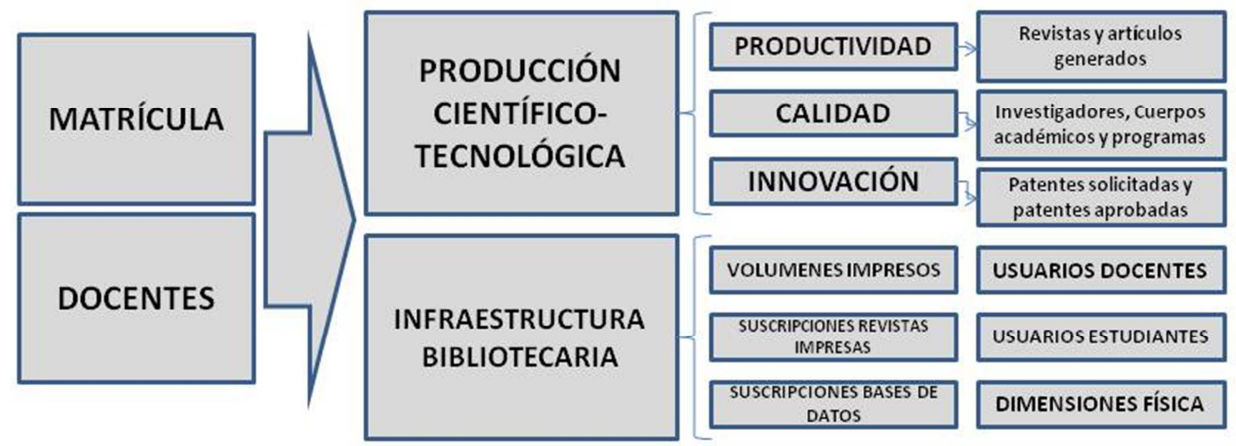
d) Se analizaron los datos en Statistical Package for the Social Sciences (SPSS), haciendo primero una revisión individual de los dos grupos de datos (infraestructura bibliotecaria y producción científica) y posteriormente, una combinación estadística de variables para determinar la influencia y correlación entre ambas.

\section{ANÁLISIS DE RESULTADOS}

Los resultados se integran en cuatro grupos: análisis estadístico de variables de matrícula de estudiantes y profesores; análisis de indicadores de infraestructura bibliotecológica; análisis de indicadores de productividad, calidad e innovación; análisis de los resultados globales y correlación de variables. Cada uno de los grupos está representado por gráficas, cuya información explícita amerita menor explicación teórica.

\subsection{ANÁLISIS ESTADÍSTICO DE VARIABLE: MATRÍCULA Y PROFESORES}

Las universidades participantes en el estudio observan un amplio espectro de matrícula, que va desde la identificación de universidades consideradas de tamaño grande (Universidades de Guadalajara, Nuevo León, Veracruz, Puebla, Sinaloa y Estado de México) que superan los 40 mil estudiantes hasta el límite de los cien mil inscritos en niveles técnico (si lo hubiera), licenciatura y posgrado, siendo el otro extremo de la escala las instituciones de educación superior identificadas dentro de la categoría de universidades chicas (que apenas alcanzan los $15 \mathrm{mil}$ estudiantes), en donde se identifican 14, siendo la de menor población El Colegio de Sonora con 73 estudiantes (figura 2).

Tan amplio es el espectro de matrícula registrada en cada una de las universidades evaluadas, que es tomado como una condición para iniciar el análisis en relación con datos ponderados sobre la base de número de profesores principalmente. Posteriormente se desarrollaron comparaciones de variables, demostrándose que en ocasiones la relación de la matrícula registrada por institución no es garantía en la proyección de infraestructura informativa, ni tampoco con los niveles de producción científica. 
Figura 2. Distribución de matrícula por universidades

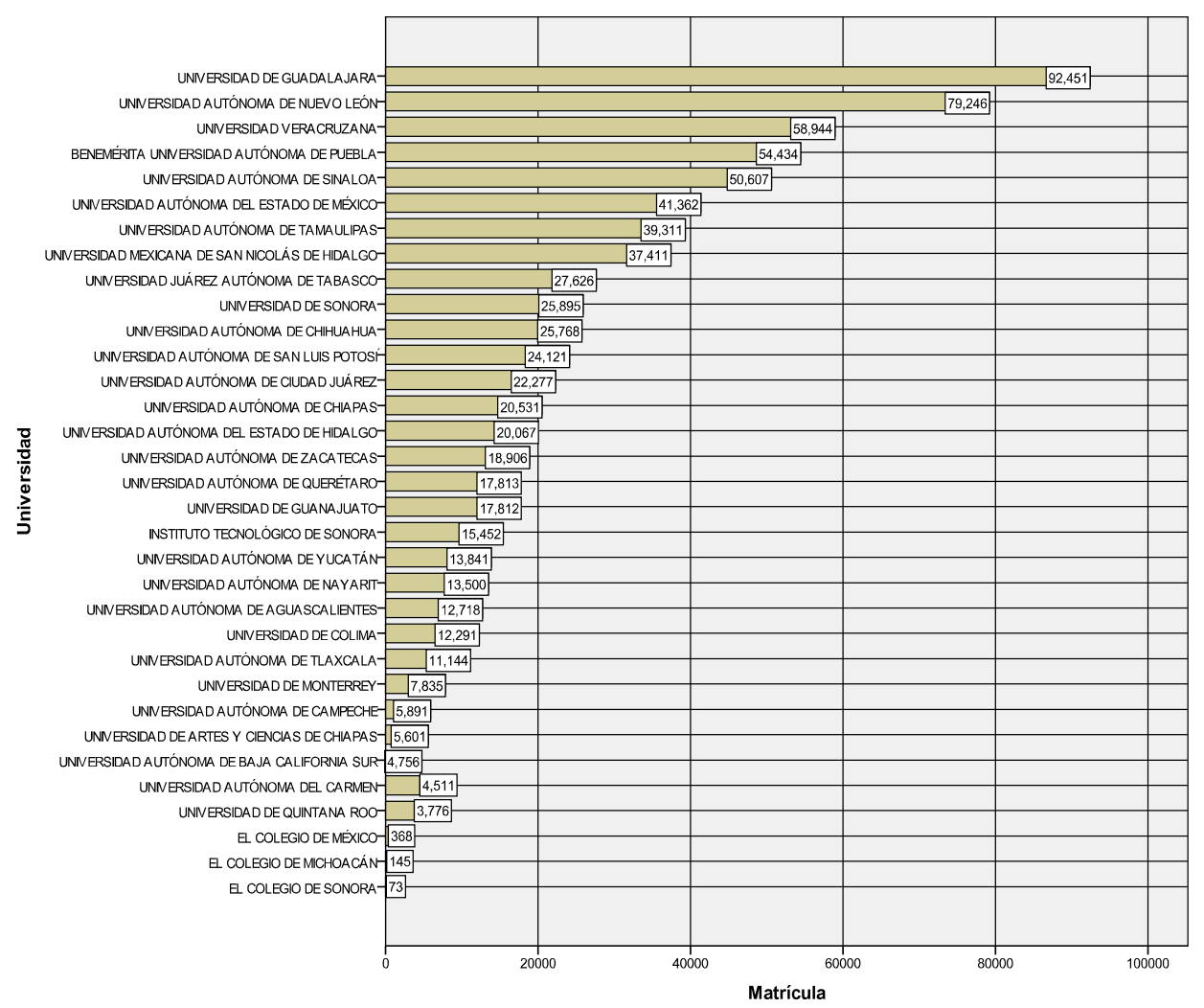

En México, uno de los parámetros para evaluar la calidad de las universidades en cuanto a sus niveles de productividad, es considerar que aquellas que atienden mayor matrícula con menor cantidad de docentes son las que mejor indicadores ofrecen. Sin embargo, de acuerdo a la relación de número de alumnos por profesor (figura 3), puede observarse que universidades, como es el caso de El Colegio de México, apunta todo lo contrario, ya que con una relación más baja de alumnos por profesor presenta indicadores de productividad y calidad científica representativamente altos.

Caso contrario sucede con universidades que no se distinguen por presentar indicadores suficientes de productividad y calidad científica representada en generación de productos publicables, sin embargo, sí muestran suficiencia en los indicadores en el ámbito de relación de alumnos atendidos por docentes, lo que se proyecta en que la productividad científica no se refleja en resultados favorables. 
Figura 3. Promedio de alumnos atendidos por profesor

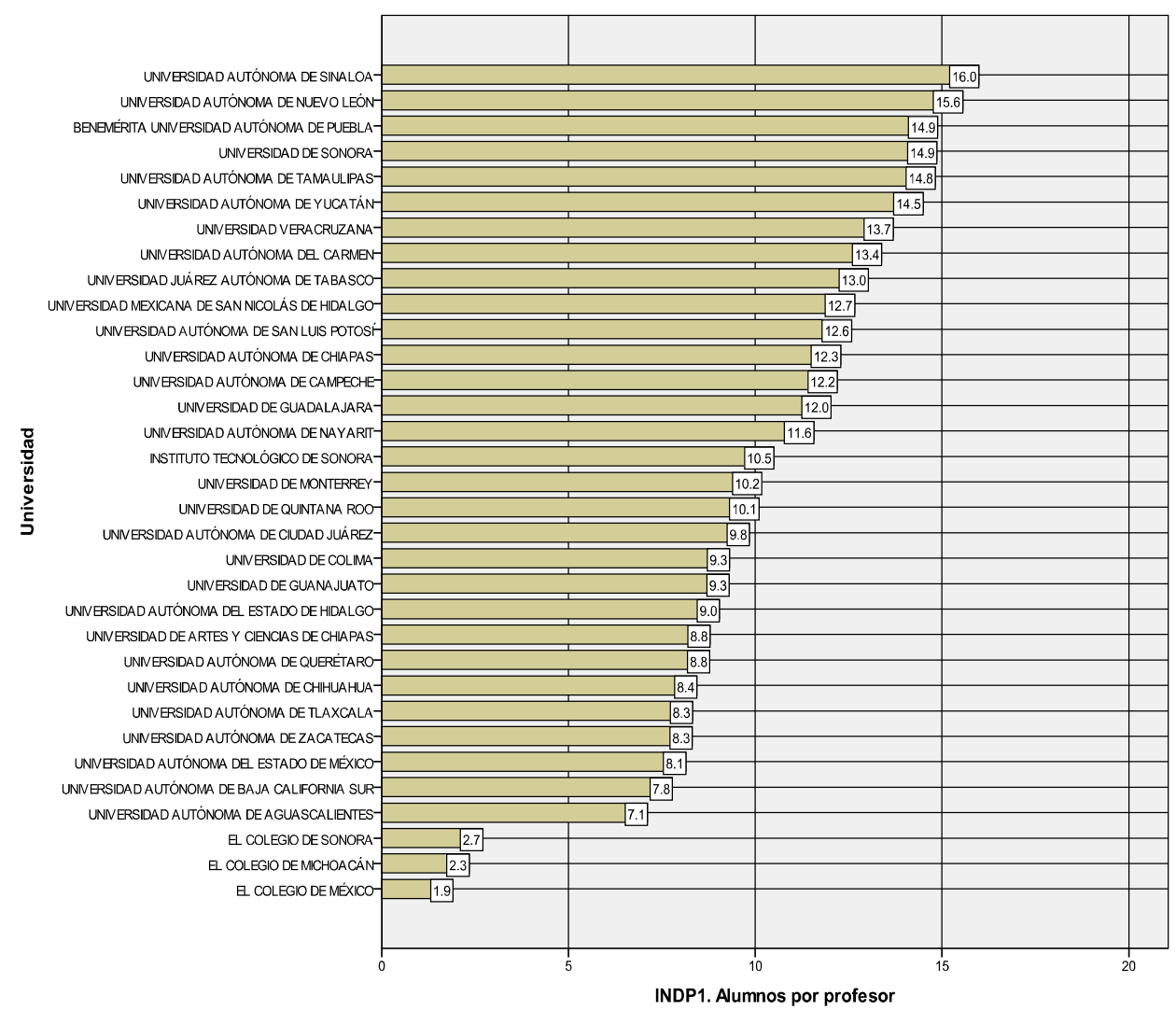

Reforzando el paradigma antes descrito en relación a la concepción que se tiene en México de que la productividad de las universidades debe medirse, considerando la importancia del parámetro de que a menor matricula atendida por profesor mejor productividad, el diagrama de dispersión (figura 4), muestra que la dotación de personal académico se determina por la cantidad de alumnos que se atienden, más no por la producción científico-tecnológica.

Lo dramático de los resultados es que El Colegio de México (2) atiende a una población mínima promedio por profesor (1.9) y su productividad, expresada posteriormente, es muy alta. Tales resultados no tienen el mismo efecto, al analizar que la Universidad de Guadalajara (26), Universidad Autónoma de Nuevo León (13), Universidad Veracruzana (33) y Universidad Autónoma del Estado de México (23), siendo las más grandes en población estudiantil atendidas, en forma proporcional, por menos docentes, no garantizan altos niveles de producción científico-tecnológica. La consulta específica de la posición que ocupan el resto de 
las instituciones de acuerdo al número que las identifica puede consultarse en la lista de instituciones del anexo 1.

Figura 4. Diagrama de dispersión de promedio de matrícula atendida por docentes

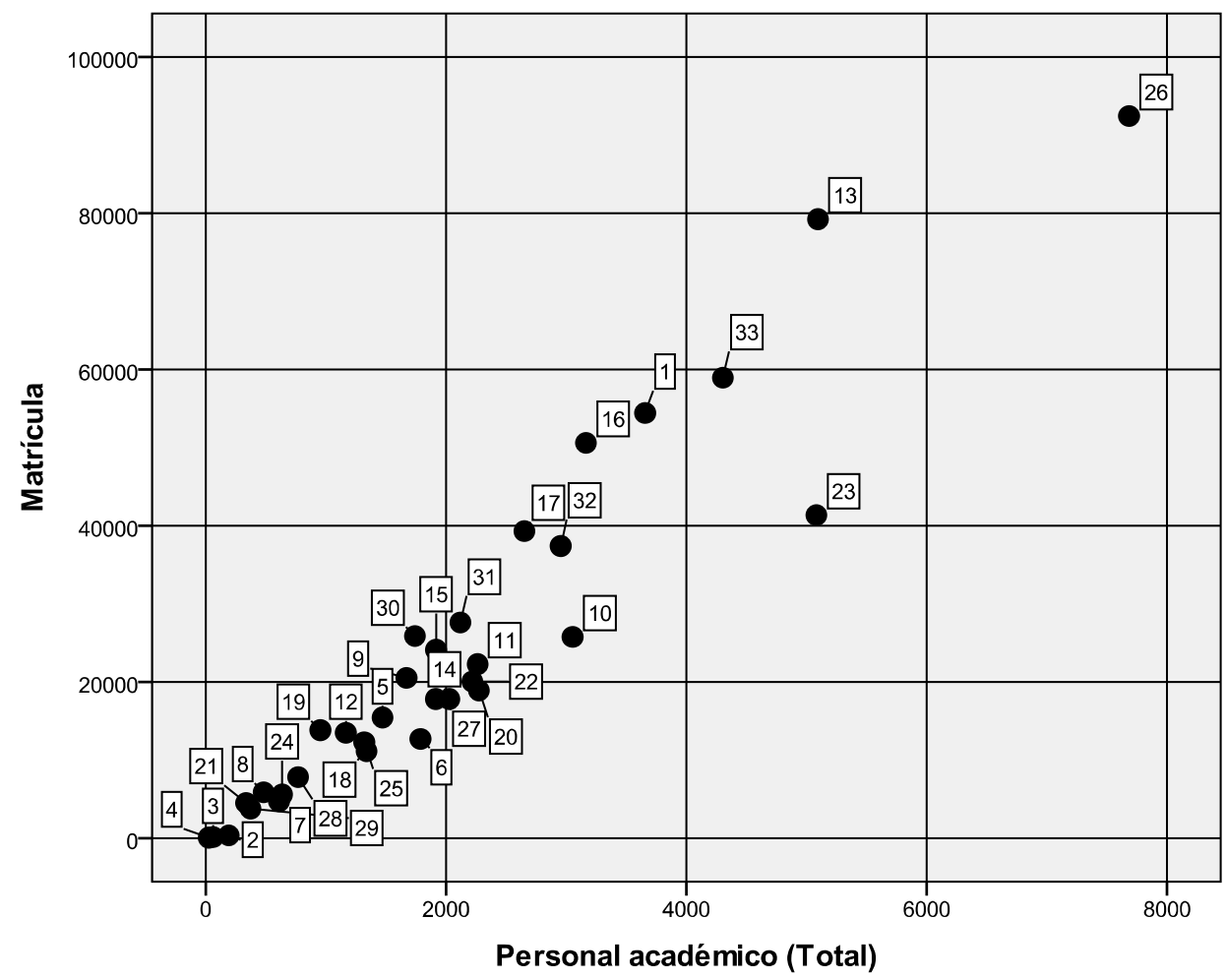

\subsection{ANALISIS DE INDICADORES DE INFRAESTRUCTURA BIBLIOTECARIA}

Este estudio maneja seis indicadores de recursos informativos vinculados con la infraestructura bibliotecaria, los cuales fundamentalmente se refieren a elementos físicos que facilitan el uso y acceso a los recursos documentales. Considera colecciones: de volúmenes y de suscripciones a revistas, ambas impresas; acceso a documentos electrónicos de acuerdo a la disposición de bases de datos; respecto a los usuarios, incluye a estudiantes y a docentes-investigadores; y, superficie física de las instalaciones bibliotecarias en metros cuadrados.

Tal como se menciona con anterioridad, la dispersión en el tamaño poblacional de estudiantes y profesores en las universidades participantes es marcado, razón por la cual se ponderan datos sobre la base de unidades fijas de comparación. Por ejemplo, para el indicador de número de volúmenes (figura 5), se analizaron los 
datos tomando como referencia el promedio de volúmenes por profesor, observándose que universidades de tamaño chico, como los Colegios de Michoacán, de México y de Sonora presentan los indicadores más relevantes, siendo que las instancias evaluadas de tamaño mediano y grande, muestran indicadores bajos.

Figura 5. Número de volúmenes impresos por profesor

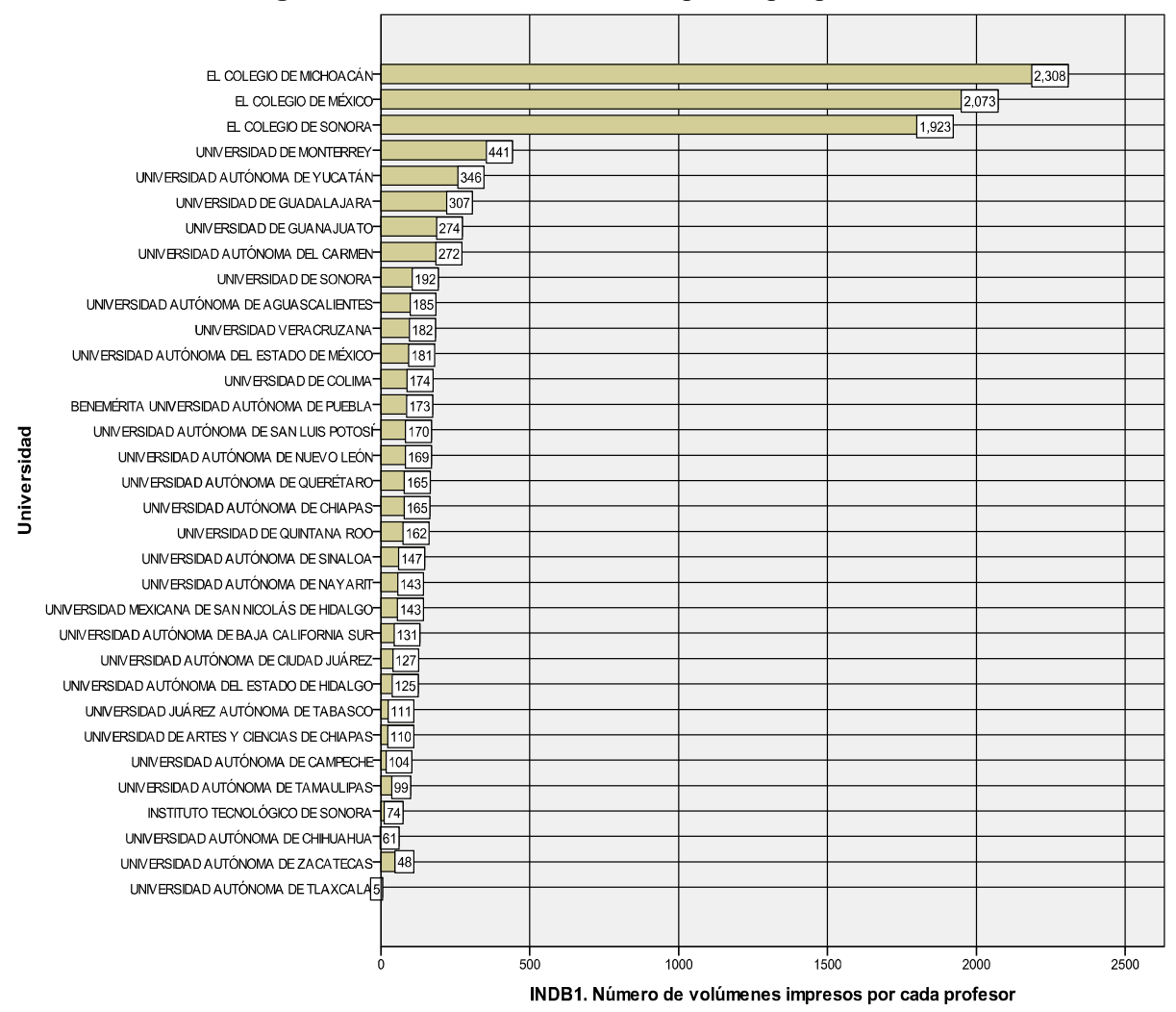

En relación con el indicador de evaluación de la infraestructura bibliotecaria, definido como el número de suscripciones a revistas impresas, es importante indicar que algunas de las universidades participantes manifestaron haberle dedicado menor importancia en los últimos años, debido esto al acceso a bases de datos, que facilitan en gran medida, el acceso a mayor número de documentos sin necesidad de mantener colecciones físicas.

En este indicador, se analizan los datos usando una ponderación de datos sobre la base de cada 100 profesores en relación con el número de suscripciones a revistas (figura 6), observándose la constante de resultados positivos en El Colegio de de Sonora, El Colegio de Michoacán y El Colegio de México, así también, se incluye 
en el grupo a la Universidad de Monterrey, la cual es de financiamiento privado y de tamaño chico. Aún cuando algunas universidades observan datos medios, la gran mayoría muestran promedios bajos en la disposición de publicaciones seriadas en formato físico como parte de su infraestructura bibliotecaria.

Figura 6. Número de suscripciones a revistas impresas

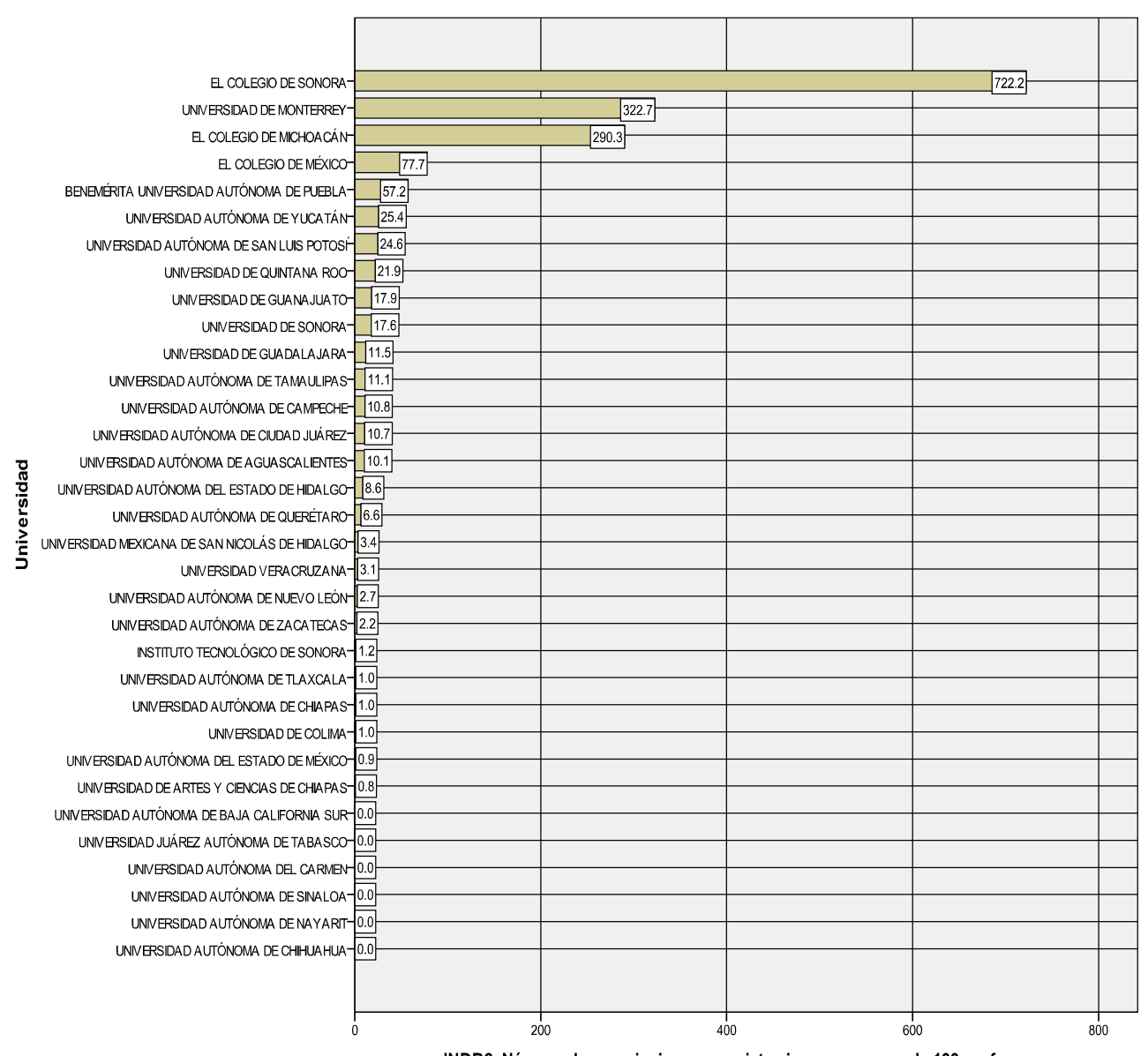

INDB2. Número de suscripciones a revistas impresas por cada 100 profesores

El tercer indicador vinculado a la evaluación de la infraestructura bibliotecaria, registra el número de suscripciones a bases de datos (ponderadas sobre la base de cada 100 profesores), en donde, El Colegio de Sonora muestra los datos más altos, seguido por El Colegio de México, El Colegio de Michoacán y la Universidad de Baja California Sur. El resto de las universidades evaluadas ofrecen datos bajos y poco representativos. 
Figura 7. Número de suscripciones a bases de datos

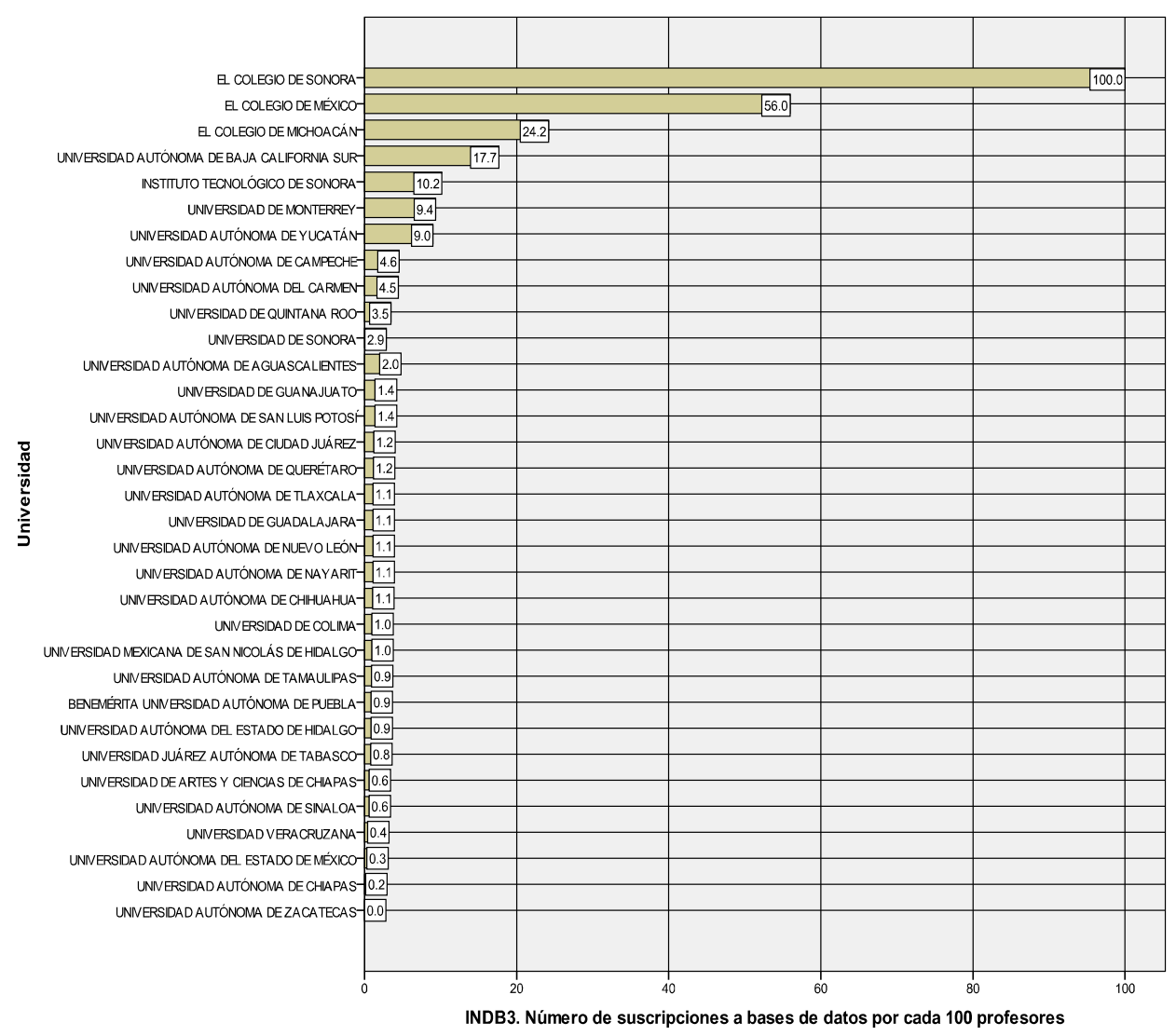

El indicador de número de usuarios docentes, cuarto en su tipo en relación con la evaluación de la infraestructura bibliotecaria, evalúa la frecuencia en el uso de recursos informativos por parte de los académicos (figura 8). Aunque la constante en otros indicadores previos favorecen fundamentealmente a los Colegios de Michoacán, de Sonora y de México, en este indicacador muestra que el uso de los recursos informativos por parte de los docentes e investigadores son bajos, poniéndose a la cabeza: la Universidad de Monterrey, la Benemérita Universidad Autónoma de Pueba y la Universidad de Nayarit. El resto de las instituciones mantienen la constante de poca afluencia de docentes e investigadores como usuarios de los recursos bibliotecarios. 
Figura 8. Número de usuarios docentes

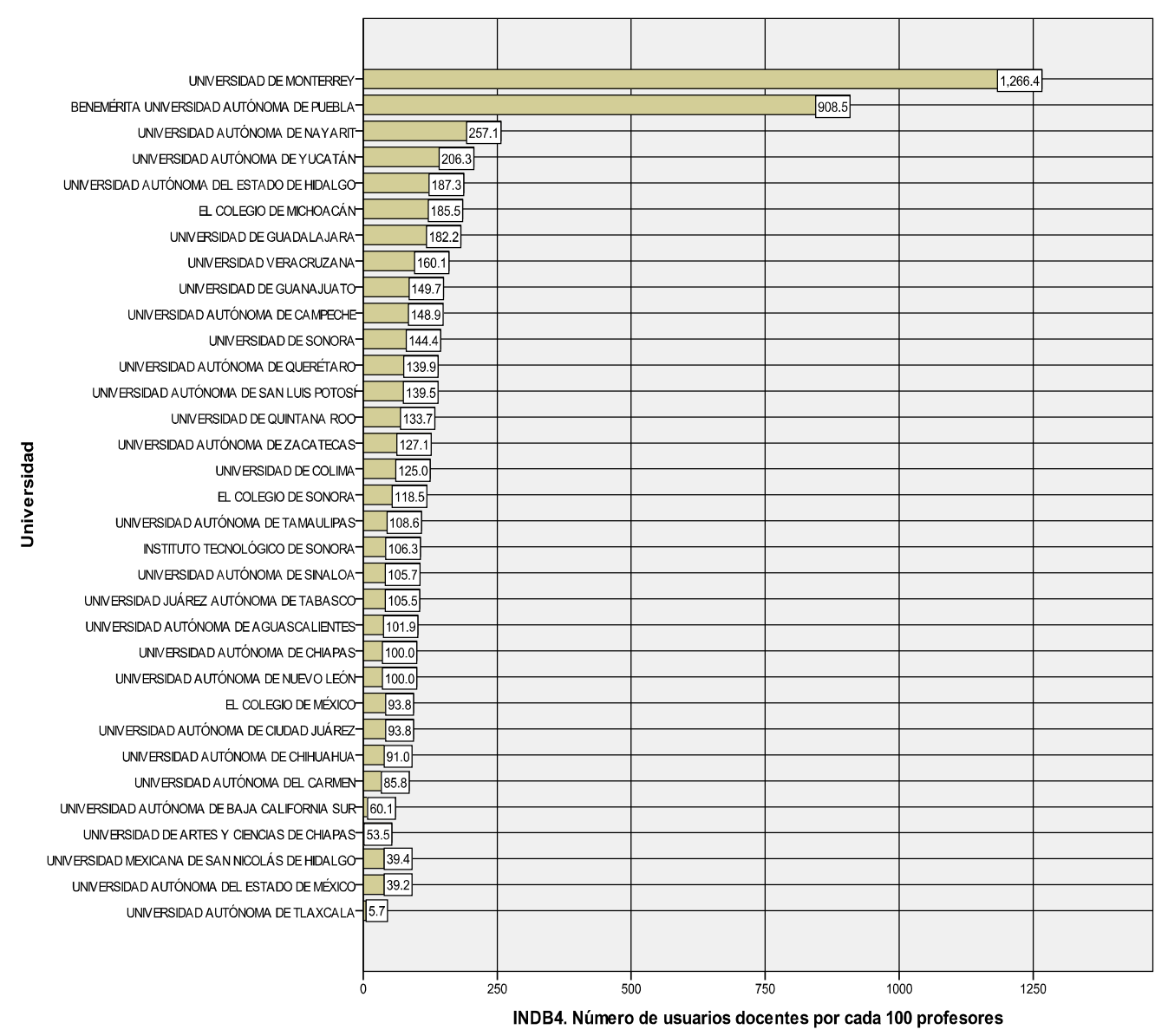

Además de los docentes e investigadores, el otro grupo de usuarios incluidos dentro del rubro de indicadores sobre infraestructura bibliotecaria, son los estudiantes, quienes cabe considerar que muestran un comportamiento en sus regularidades al disponer de los recursos informativos, animados por su propia actividad académica y a quienes se les reconoce cierto patrón de comportamiento, partiendo de las actividades curriculares que los docentes imponen dentro de sus clases como parte de los procesos de enseñanza-aprendizaje.

En este indicador no se miden las características o condiciones de uso de los recursos informativos, cuya consulta puede estar vinculada al mero consumo de información y no necesariamente a sustentar procesos de investigación. Los resultados observados (según la figura 9), muestran los resultados más representativos: la Universidad de Artes y Ciencias de Chiapas, la Benemérita Universidad Autónoma de Puebla y la Universidad de Monterrey. Aquí es importante resaltar que los indicadores 
menos representativos, recaen en El Colegio de México, El Colegio de Sonora y El Colegio de Michoacán, instituciones educativas que en otros indicadores apuntalaron el comportamiento más representativo.

Figura 9. Número de usuarios estudiantes

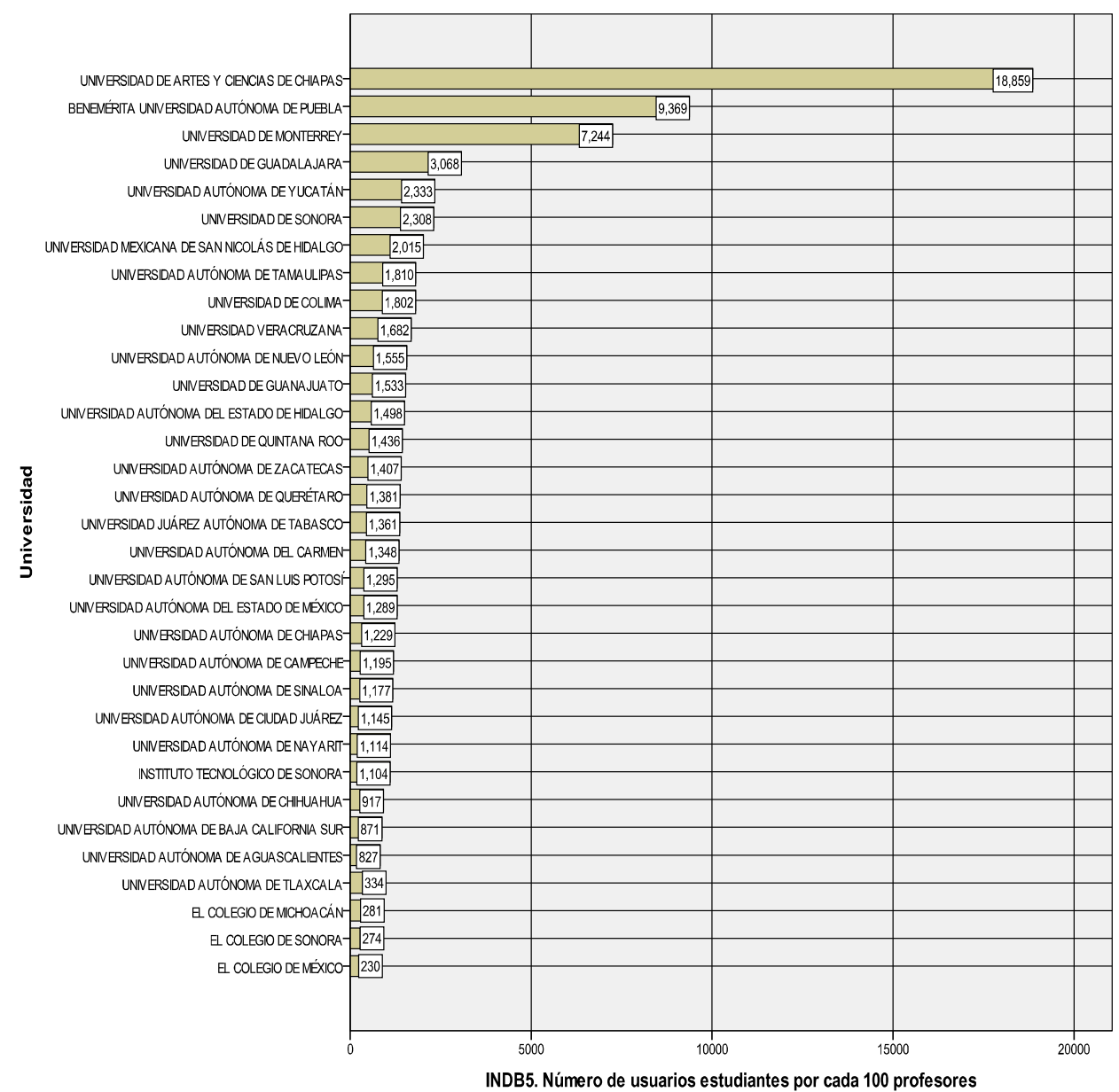

El sexto indicador, infraestructura bibliotecaria, se midió por el número de metros cuadrados de espacio físico destinado a los ambientes vinculados con los recursos informativos. Sobresalen los resultados de El Colegio de México, institución que destina el mayor número de metros cuadrados en espacios bibliotecarios por cada 100 profesores $(37,916)$, y del resto de las universidades evaluadas, la más alta alcanza sólo el 10\% de lo destinado por la más sobresaliente (Universidad de Colima con 3,788), tal como se puede comparar en la figura 9. 
Figura 10. Número de metros cuadrados de infraestructura física bibliotecaria

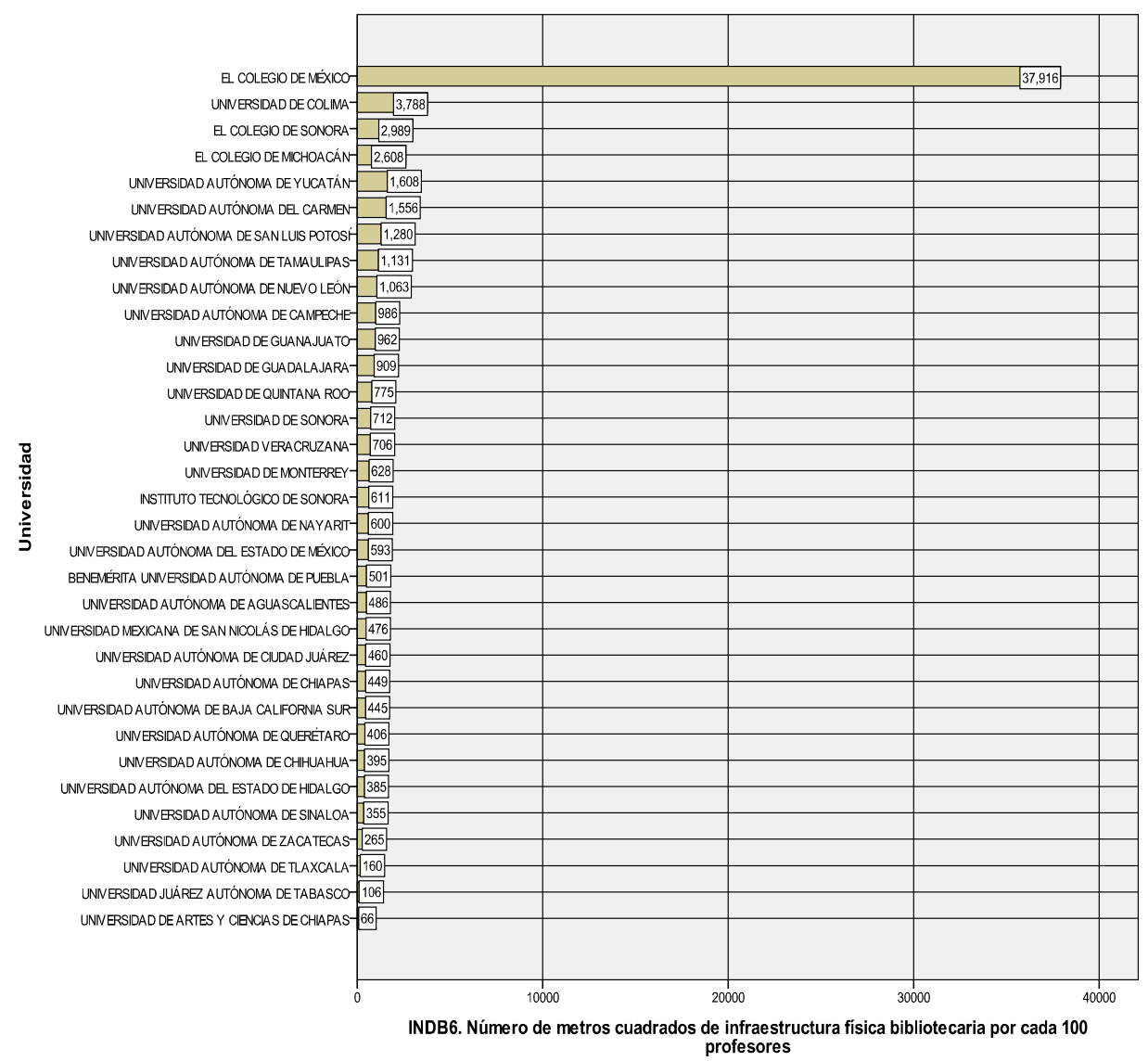

\subsection{INDICADORES DE PRODUCTIVIDAD}

La productividad de las universidades en el renglón de la producción científico tecnológica está determinada en este estudio por el número de publicaciones generadas por la misma institución y por el número de artículos publicados vertidos de los docentes e investigadores de las propias instituciones.

De acuerdo al análisis del indicador relacionado con la generación de medios de divulgación como primer parámetro de evaluación de la productividad en las universidades, entendido este como la disposición institucional a crear publicaciones de calidad propias (revistas científicas), las cuales pueden ser un medio de inclusión de publicaciones de docentes-investigadores de la misma institución como de otras, se desarrolló un análisis sobre el número de publicaciones incluidas en los catálogos de Latindex (Sistema Regional de 
Información en Línea para Revistas Científicas de América Latina, El Caribe, España y Portugal, creado por la UNAM) y en el Padrón de Publicaciones de Excelencia del CONACYT (el cual es el órgano más importante en relación con los aspectos de producción científica en México), se consideraron los datos en relación con el número de publicaciones (títulos) por cada 100 profesores cuyos resultados se presentan en la figura 10 .

Según estos resultados, nuevamente se observa que la cantidad de profesores adscritos a una universidad no es determinante sobre el número de publicaciones de generación interna. Por ejemplo, las instituciones mejor evaluadas son: El Colegio de México es la institución que mejores indicadores muestra (11.92 publicaciones por cada 100 profesores), El Colegio de Sonora (11.11) y el Colegio de Michoacán (8.06) y las 30 universidades restantes tienden a desarrollar pocas publicaciones, siendo el espectro de 1.47 publicaciones por cada 100 profesores en la Universidad de Yucatán como el más alto y de ninguna publicación en las Universidades de Baja California Sur y Guanajuato.

Figura 11. Número de publicaciones generadas por universidades incluidas en Latindex y CONACYT

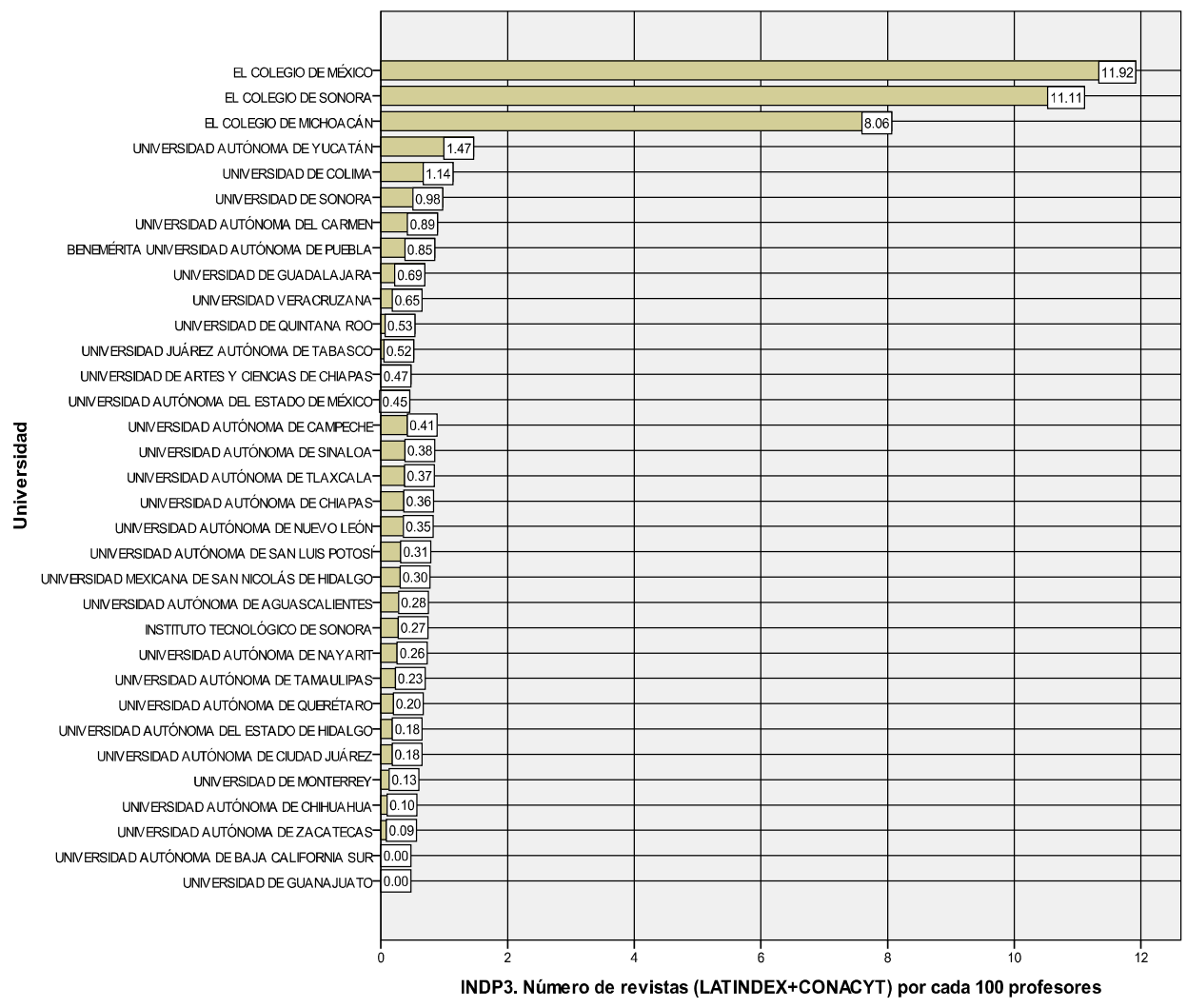


El segundo parámetro de evaluación de la productividad de las universidades, está relacionado con la generación artículos científicos incluidos en revistas reconocidas por el ISI, SCOPUS (base de datos bibliográfica y de citación para artículos en revistas académicas editada por Elsevier), PERIODICA (Índice de Revistas Latinoamericanas en Ciencias) y CLASE (Citas Latinoamericanas en Ciencias Sociales y Humanidades) ambas creadas por la UNAM, promediando los resultados globales arrojados durante el 2011. Aunque cada una de las bases de datos representa diversos niveles de exigencia y ámbitos en la publicación de artículos, puede considerarse que ISI entra dentro del parámetro de revistas indizadas o indexadas y las tres restantes relacionadas con la inclusión de revistas arbitradas o peer-reviewed.

Los datos considerados en la figura 11, representa los altos niveles de productividad en generación de conocimiento durante el 2011 por parte de El Colegio de México (52.8 artículos por año evaluado por cada 100 profesores), la Universidad Autónoma de Yucatán (40.1 artículos por año evaluado por cada 100 profesores), El Colegio de Michoacán (33.9 artículos por año evaluado por cada 100 profesores) y El Colegio de Sonora (33.6 artículos por año evaluado por cada 100 profesores), siendo que en el otro extremo, se observa al Instituto Tecnológico de Sonora y a la Universidad Autónoma de Ciudad Juárez con 2.9 y 2.6 artículos por año evaluado por cada 100 profesores. En el rango de los 20 a 30 artículos publicados se identifican tres universidades, de 10 a 20 fueron 11 universidades y 13 universidades por debajo de los 10 artículos producidos en el año evaluado. 
Figura 12. Número de artículos publicados en ISI, SCOPUS, PERIODICA y CLASE

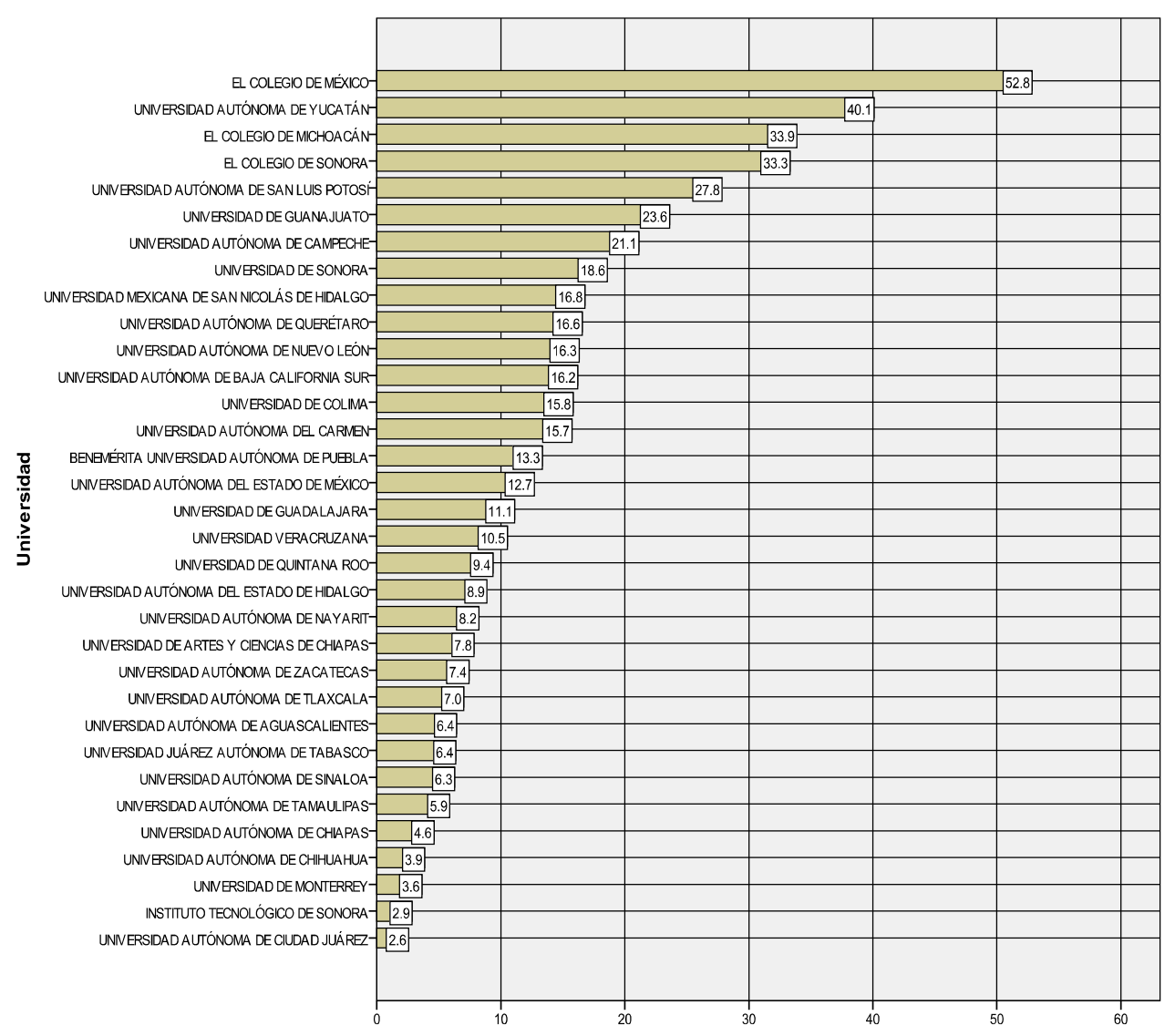

INDP2. Número de artículos (ISI+SCOPUS+PERIODICA+CLASE) por cada 100 profesores

\subsection{INDICADORES DE CALIDAD}

Para efectos de este estudio, la evaluación de la calidad de las universidades participantes estará determinada por tres indicadores:

a) Número de profesores e investigadores reconocidos por el SNI, órgano mexicano dedicado a reconocer la labor de las personas dedicadas a producir conocimiento científico y tecnológico, cuyo requisito de ingreso está determinado por los grados académicos (doctorado) y la producción científica, por mencionar los principales.

b) Número de cuerpos académicos o grupos de trabajo colegiado reconocidos por el Programa de Mejoramiento del Profesorado (PROMEP), entidad del gobierno federal mexicano que incentiva la creación de cuerpos colegiados hacia la producción científica. 
c) Número de programas académicos acreditados por la Comisión Interinstitucional para la Evaluación de la Educación Superior (CIEES), Programa Nacional de Posgrados de Calidad (PNPC) y Consejo Nacional para la Acreditación de la Educación Superior (COPAES). Las tres instancias determinan en México los niveles de calidad de los programas académicos de licenciatura y posgrado.

El indicador de calidad respecto al número de profesores pertenecientes a SNI, con el fin de ponderar los resultados de forma más equitativa, fue calculado sobre la base de cada 100 profesores que integran la totalidad de la población docente en cada universidad. Destacan los resultados de El Colegio de México (89.1), El Colegio de Michoacán (79) y El Colegio de Sonora (59.3), quienes fueron los que más alto puntaje obtuvieron (figura 13).

Es importante indicar en la medición de este indicador en México, que la medición de miembros del SNI es hecho en razón del total de los docentes que participan en las universidades, sin diferenciar su condición laboral (tiempo parcial y tiempo completo), teniendo mayores posibilidades de pertenencia los segundos. En el caso de las instituciones ponderadas más favorablemente en este indicador, existe una fuerte tendencia hacia la inclusión mayoritaria de profesores de tiempo completo.

Figura 13. Número de miembros en el SNI

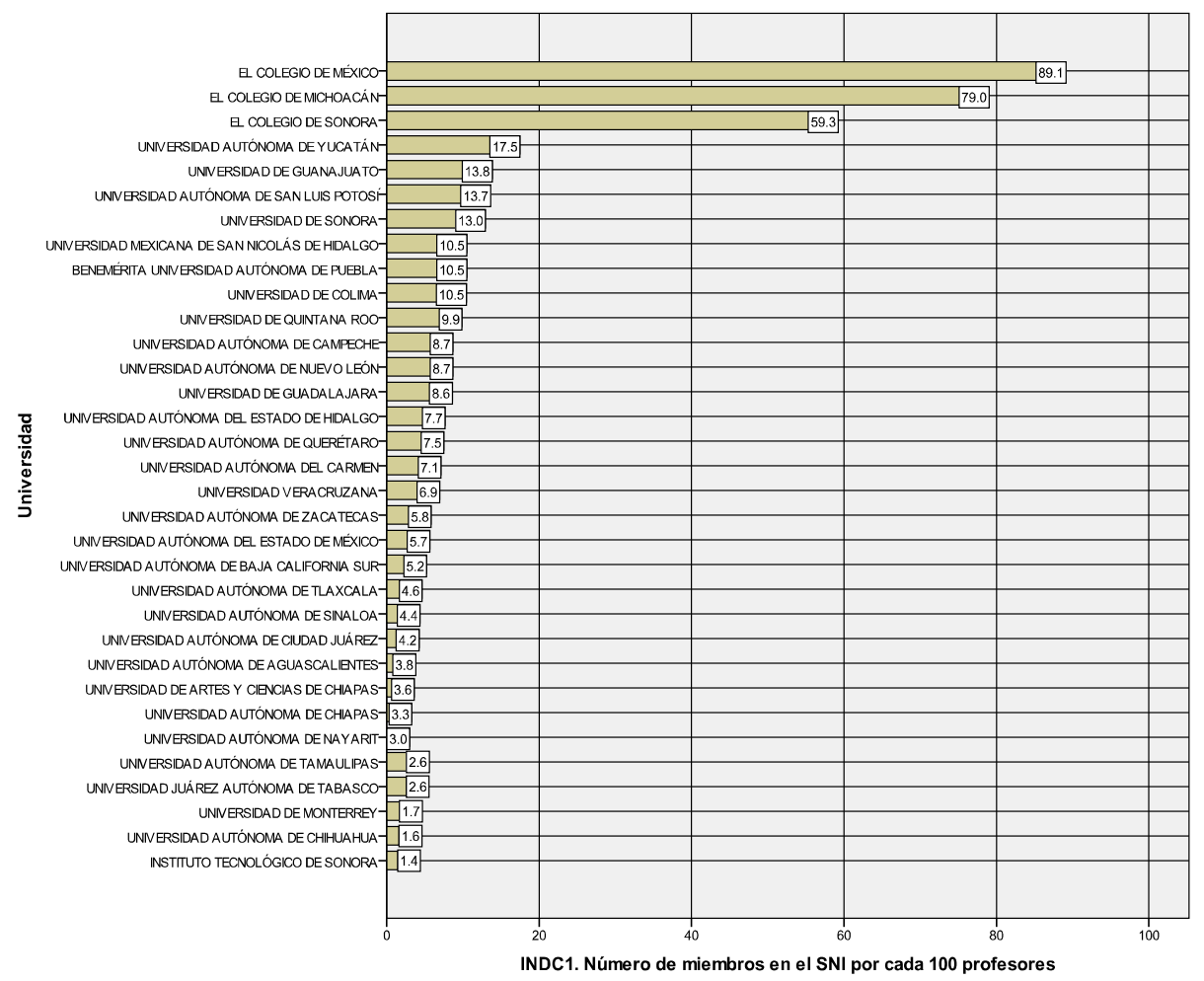


El trabajo colegiado, medido por el número de cuerpos académicos reconocidos por PROMEP, denota la posibilidad que observan las instituciones de agrupar docentes e investigadores con el fin de desarrollar trabajo colegiado, el cual se espera sea mayor en calidad y cantidad dado el involucramiento de equipos multidisciplinarios. Este indicador favorece a El Colegio de México y El Colegio de Sonora como las instituciones más sobresalientes; la universidad que observa datos menos favorables es la Universidad de Monterrey, haciendo la aclaración de que este tipo de iniciativas aplica únicamente en universidades públicas y no en privadas (figura 14).

Figura 14. Número de cuerpos académicos reconocidos por PROMEP

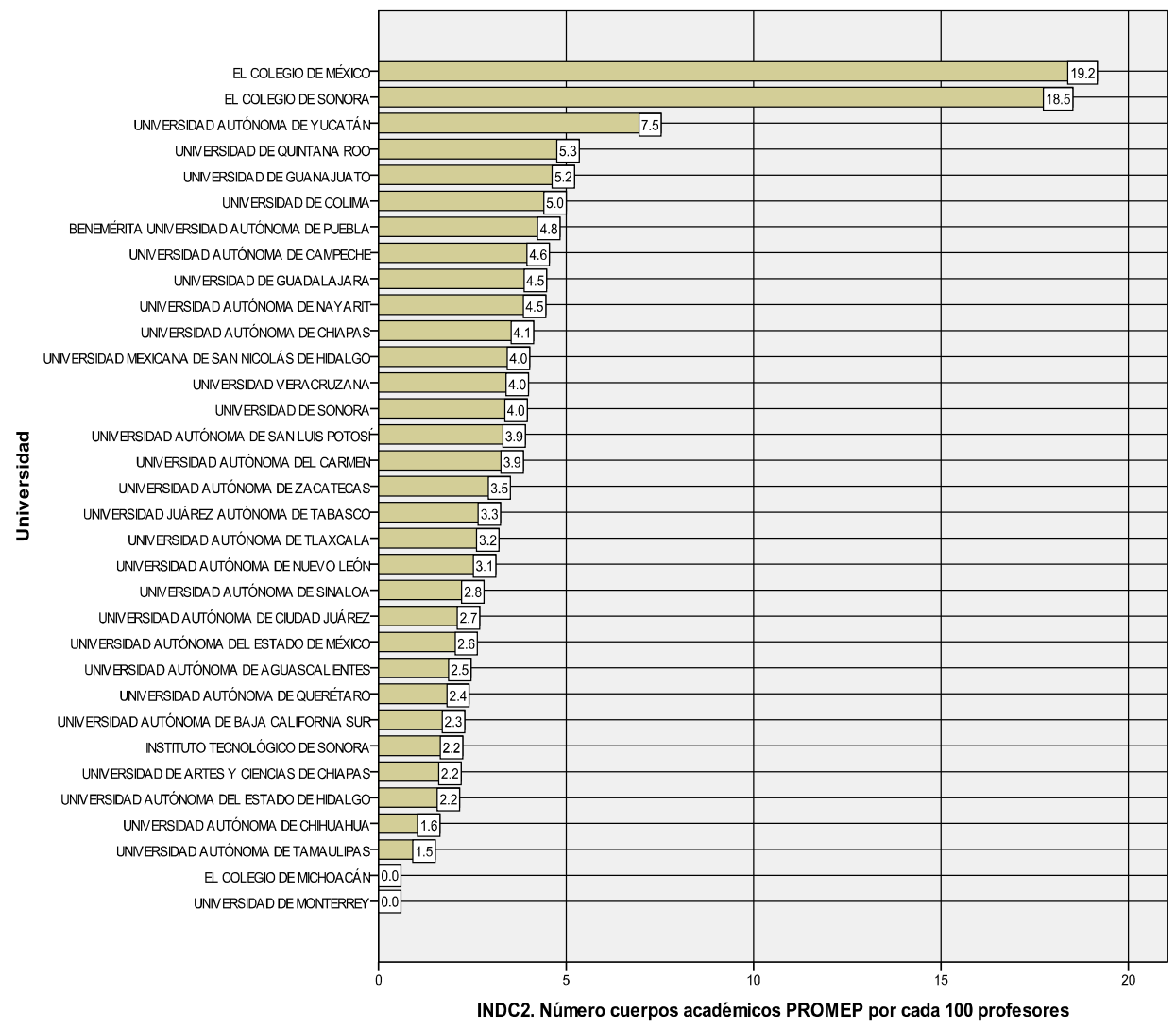

La ponderación del indicador del número de programas acreditados por CIEES, PNPC y COPAES fue hecha sobre la base de cada 10 programas académicos que ofrecen las universidades evaluadas y representando un promedio de las tres instancias evaluadoras. Este indicador determina que los organismos evaluadores observan una serie de parámetros de calidad relacionados con diversos aspectos 
tales como: niveles de formación de la planta docente, niveles de reconocimiento académico y niveles de titulación y graduación, por lo que muestran una relación alta con los aspectos de trabajo individual y colegiado que se proyecta en las condiciones de producción científica.

De acuerdo a la figura 15, la universidad que proporcionalmente ofrece mayor número de programas académicos acreditados es la Universidad Veracruzana, observándose una degradación de resultados de forma paulatina en el resto de las instituciones participantes, hasta llegar a la Universidad de Artes y Ciencias de Chiapas con el menor indicador.

Figura 14. Número de programas acreditados por CIEES, PNPC y COPAES

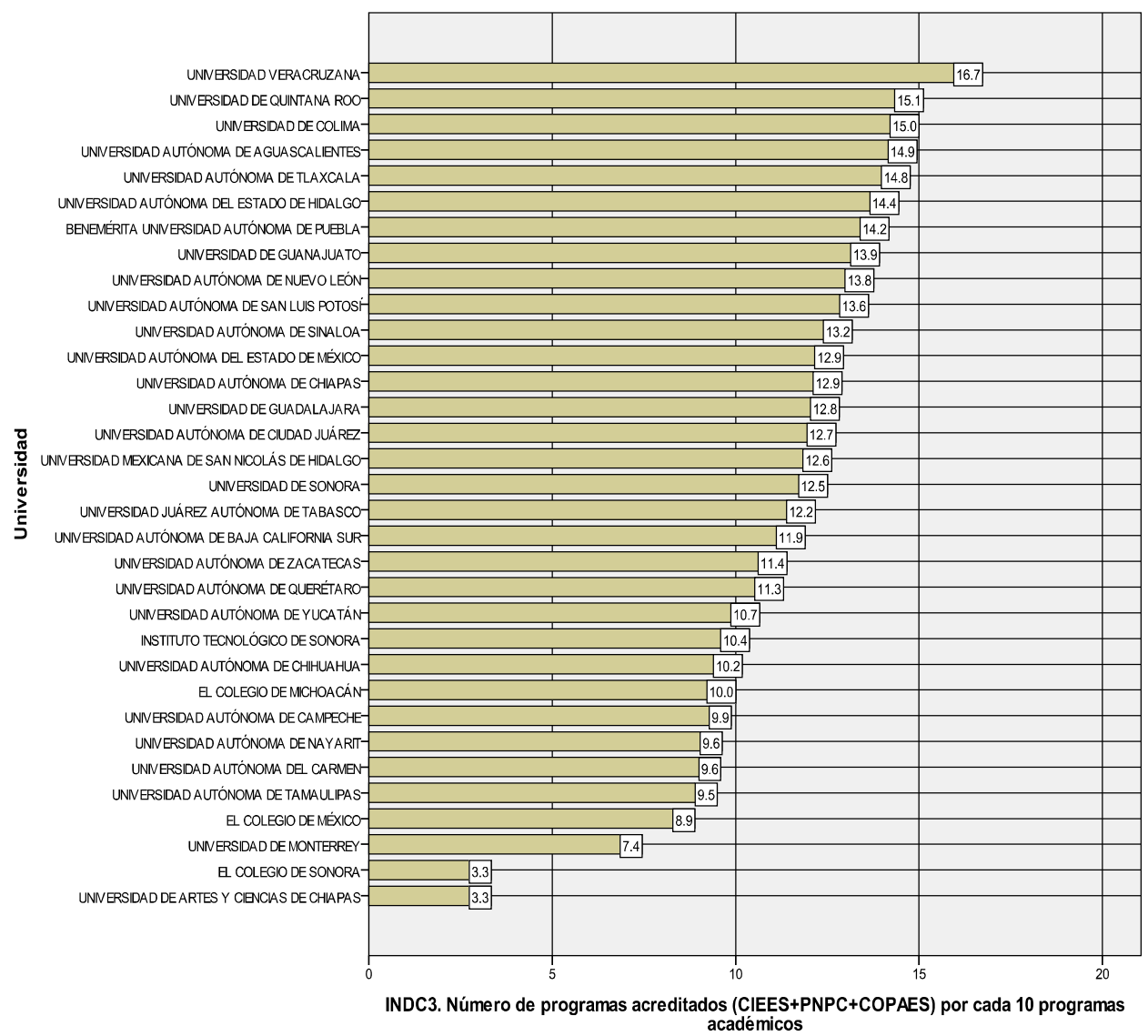

\subsection{INDICADORES DE INNOVACIÓN}

Este indicador incluye dos elementos: el primero, número de patentes solicitadas y el segundo, número de patentes aprobadas. Según los resultados 
observados en la figura 15, las universidades que destacan en la solicitud y aprobación de patentes son principalmente la Universidad de Guanajuato, Universidad Autónoma de Yucatán, la Universidad de Nuevo León y la Universidad de Colima (figura 15).

Resulta interesante inferir que la mayoría de las universidades no aportan patentes como parte de los elementos de su producción científica (18 universidades), considerándose la necesidad de identificar además otros indicadores de innovación, ya que, medirlo únicamente sólo por patentes ofrece resultados no relevantes, esto debido a que la mayoría de las universidades mexicanas se encuentran mal posicionadas al respecto ya que no patentan prácticamente nada.

Figura 16. Número de patentes solicitadas y aprobadas

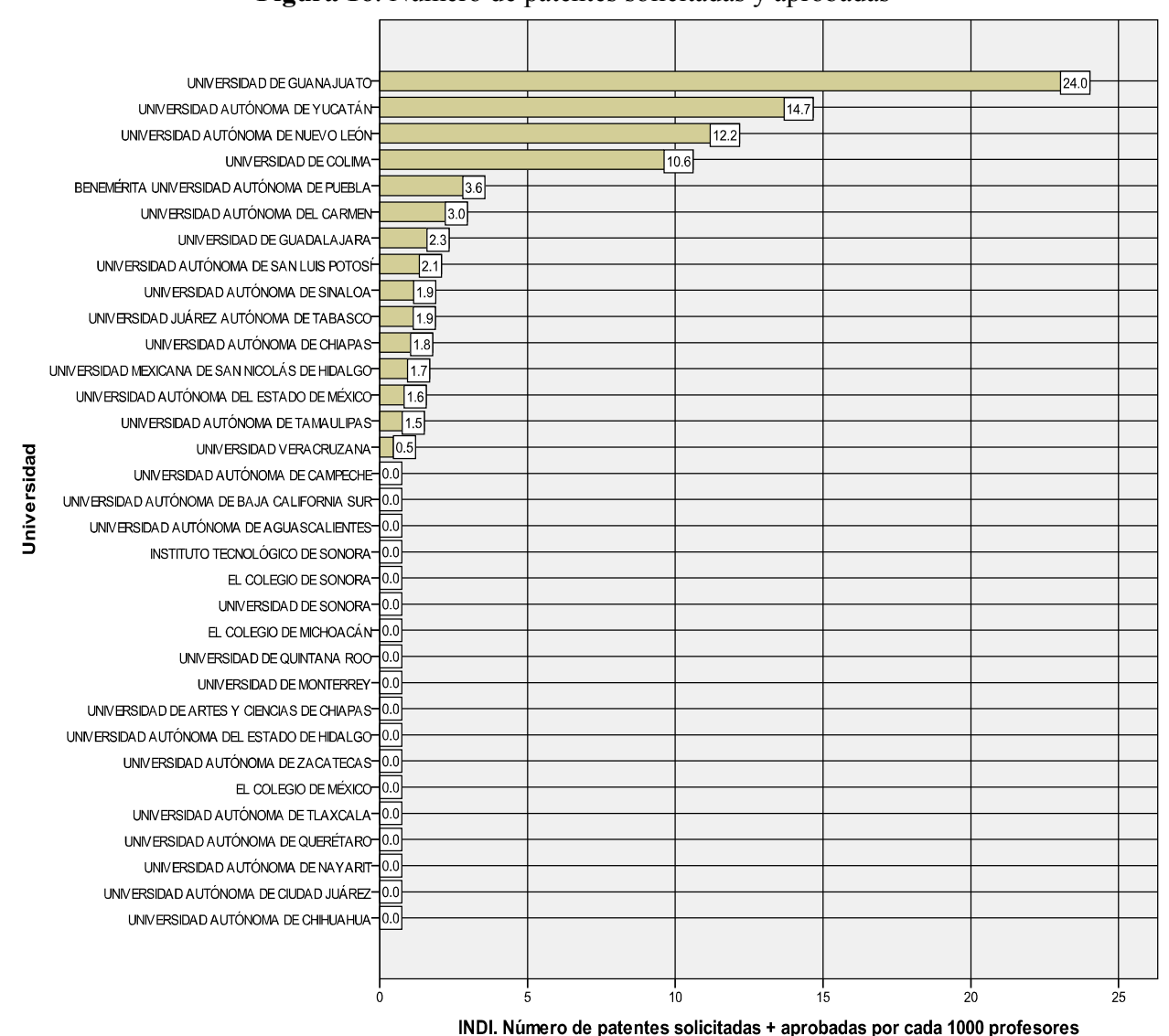




\subsection{ANÁLISIS DE RESULTADOS GLOBALES}

En este apartado se presentan los resultados globales de los tres aspectos generales de evaluación de la producción científico-tecnológica indicados dentro del modelo teórico simplificado: productividad, calidad e innovación. Es importante aclarar que el análisis de datos fue hecho con los resultados obtenidos de los indicadores finales (ponderados sobre la base un determinado número de elementos, de tal forma que permitiera hacer comparaciones más equitativas por la dispersión en número de matrícula y planta docente) y no sobre la base de los datos originales de las variables.

En cuanto a la evaluación del nivel de productividad como análisis de los resultados globales, combina los indicadores a matricula (alumnos), artículos publicados y el número de revistas científicas gestadas dentro de las propias instituciones. El resultado (figura 16), indica que en el rubro de productividad, la institución con mejor desempeño es El Colegio de México y la que observa el menor índice es la Universidad Autónoma de Chihuahua.

Figura 17. Índice de productividad de las universidades evaluadas

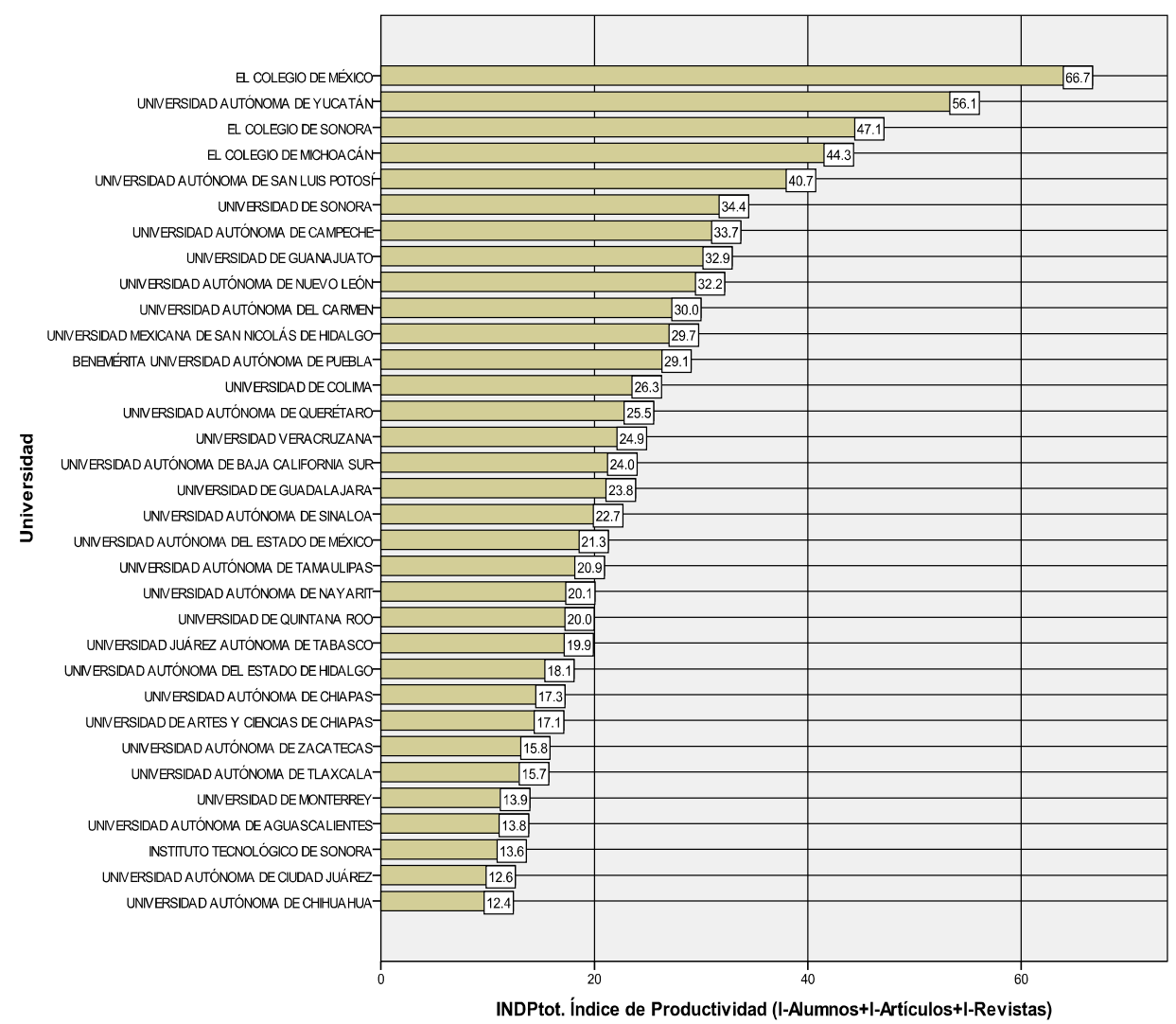


La evaluación de los resultados globales sobre calidad en la producción científica de las universidades participantes, donde se abarcan el número de docentes pertenecientes al SNI, formación de cuerpos académicos reconocidos por PROMEP y programas acreditados por las diversas instancias académicas evaluadoras, indican que las organizaciones educativas con mejores resultados son El Colegio de México, El Colegio de Michoacán y El Colegio de Sonora, en ese orden. Las 30 universidades restantes observan indicadores menores que no las colocan en el nivel de competencia en calidad con las antes mencionadas (figura 18).

Figura 18. Índice de calidad de las universidades evaluadas

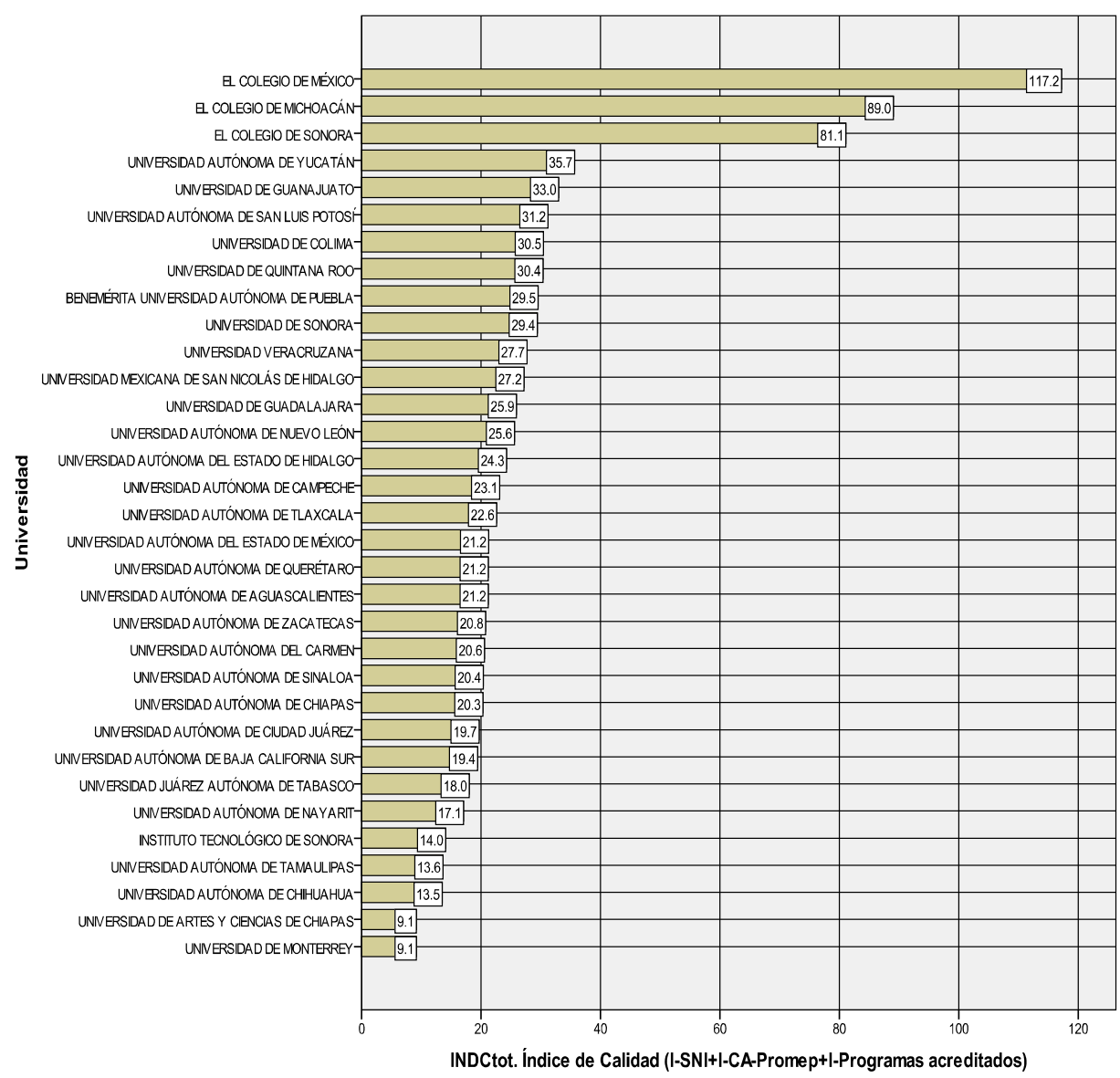

El índice de innovación como resultado global, que agrupa patentes solicitadas y aprobadas, tal como se menciona con anterioridad, resulta poco representativo para determinar de forma amplia la identificación de este aspecto de evaluación de la producción científica de las universidades, ofreciendo resultados muy similares 
a los incluidos en la figura 15, por lo tanto, se obvia la inclusión de la gráfica de resultados globales.

La integración de los resultados generales se ve culminada con una gráfica ponderada (figura 18), en la cual se incluyen los resultados de competitividad ponderada de los tres aspectos básicos de la producción científica según el modelo propuesto: productividad, calidad e innovación, de tal forma que se le da la misma importancia balanceada a los tres aspectos. Tal combinación de indicadores arroja como resultados que El Colegio de México es la universidad del grupo de entidades educativas participantes con mejor nivel de competitividad científicotecnológica, seguida por El Colegio de Michoacán, El Colegio de Sonora, la Universidad de Yucatán y la Universidad de Guanajuato, en ese mismo orden de importancia de mayor a menor.

Figura 19. Índice de competitividad ponderada

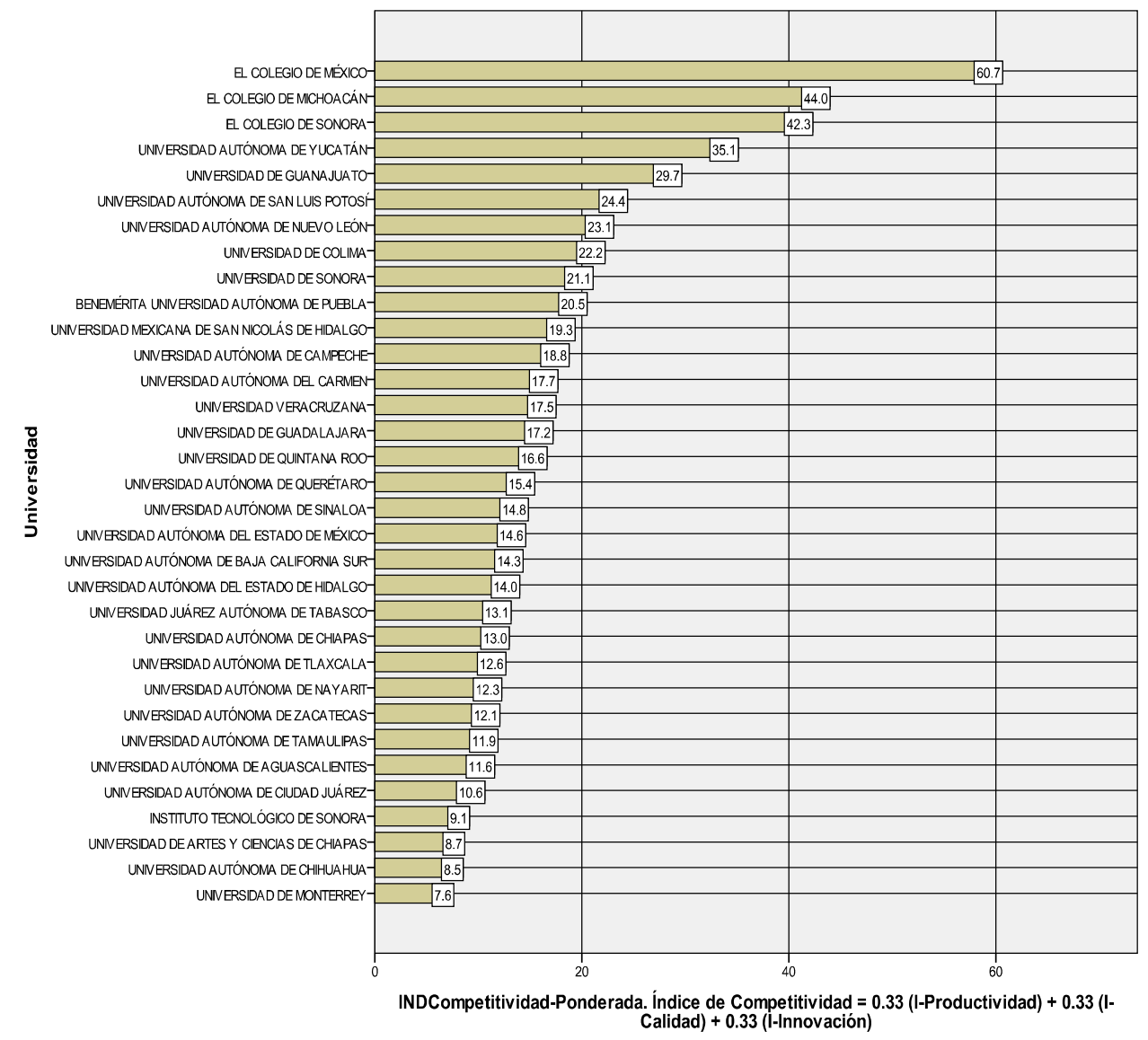


Con el fin de comparar los resultados globales de productividad científicotecnológica de las universidades incluidas en el estudio y las repercusiones que tiene la infraestructura bibliotecaria en dicho proceso de generación de conocimiento, se realizó un análisis de correlación entre ambos grupos de indicadores.

De acuerdo a los resultados arrojados de dicho análisis (anexo 2), se observó los siguientes resultados:

a) Correlación altamente significativa $(* *)$. Se presentó, en cuanto a infraestructura bibliotecaria (indicadores de número de volúmenes, número de suscripciones a bases de datos y número de metros cuadrados como superficie bibliotecaria) con los índices de productividad, calidad, competitividad y competitividad ponderada.

b) Correlación significativa $(*)$. Se presentó correlación significativa entre el índice de productividad y el número de suscripciones a revistas impresas.

c) Correlación no significativa o que no son variables que estén asociadas (correlación nula). Todos los índices de innovación con todos los índices de infraestructura bibliotecaria, así como, todos los índices de productividad científica con el número de usuarios docentes y estudiantes.

\section{CONCLUSIONES}

La producción científica en las universidades mexicanas requiere aún de mayores esfuerzos para mejorar sus indicadores, ya que los resultados observados denotan bajos niveles de investigación científica proyectada en formas de comunicación de la ciencia. Las políticas públicas relacionadas con los asuntos de generación de conocimiento no manifiestan logros importantes a través de los programas establecidos con este fin.

Las universidades en general tienen el compromiso de contribuir al desarrollo científico de los países en donde están establecidas, buscando nuevas formas que propicien la creación de conocimiento en forma sistemática. Aunque todo estudio de esta naturaleza ofrece limitantes en la generalización de los resultados que arrojan, especialmente cuando se estudian grupos de entidades formadas de manera, prácticamente, voluntarias y sin un sentido estricto de selección o de obligatoriedad.

Los hallazgos principales observados representan que los niveles de producción científica se manifiestan principalmente en universidades chicas, generalmente especializadas en niveles de posgrado y que guardan un enfoque estricto más hacia la investigación y la formación de recursos humanos de alto nivel, más que en la docencia como actividad principal.

Además, se observa que la productividad y la calidad de las universidades evaluadas, está correlacionadas de una manera altamente significativa con los elementos principales de la infraestructura bibliotecaria. Se reafirma la necesidad de identificación de nuevos elementos para la medición de la innovación científica, ya 
que el mero estudio del indicador vinculado con la generación y aprobación de patentes es insuficiente y con nula correlación con el resto de las variables.

Finalmente, es necesario un cambio de paradigma en los conceptos de medición de la productividad, ya que de acuerdo a los resultados observados, las universidades que atienden mayor número de estudiantes con menor número de docentes no son necesariamente eficientes, sin embargo, este estudio muestra que las universidades más productivas y de mayor calidad en producción científica son las que mantienen más bajos niveles de alumnos promedio atendidos por profesor. Igual se observa que a mayor infraestructura bibliotecaria, mejores resultados relacionados con los niveles de producción científico-tecnológica.

\section{REFERENCIAS BIBLIOGRÁFICAS}

ASSOCIATION OF COLLEGE AND RESEARCH LIBRARIES. SECCIÓN DE BIBLIOTECAS UNIVERSITARIAS. COMITÉ DE REVISIÓN DE NORMAS PARA BIBLIOTECAS UNIVERSITARIAS (1994). Normas para bibliotecas universitarias: Evaluación de la eficacia, preparadas por el comité de revisión de normas para bibliotecas universitarias de la sección de bibliotecas universitarias de la ACRL. Recuperado de http://dialnet.unirioja.es/servlet/articulo? codigo =50921.

BLANCO, E., CASALDÁLIGA, A. (2012). Papel de la biblioteca en la difusión de la producción científica en la Universitat Pompeu Fabra. El Profesional de la Información. 21, 627-631.

BREEDING, M. (2011). Using Technology to Enhance a Library as Place. Computers in Libraries. 31(3): 29-31.

BRYANT, B. (1989). Guide for Written Collection Policy Statements, Chicago: ALA Editions.

BUSTAMANTE RODRÍGUEZ, A.T., PADIAL LEÓN, A. (2003, julio-diciembre). Perspectiva ética y deontológica para la profesión bibliotecaria. Biblios, IV (16).

BUSTOS GONZÁLEZ, A. (2007). Bibliotecas universitarias: ¿sabemos medir sus resultados e impactos? El Profesional de la Información, 16(4): 281-286.

CABRAL VARGAS, B. (2007). El papel de las bibliotecas y la educación en la gestión de conocimiento en la sociedad contemporánea. Alexandria: Revista de Ciencias de la Información, 4 (6):15-19.

CONSEJO NACIONAL PARA ASUNTOS BIBLIOTECARIOS DE LAS INSTITUCIONES DE EDUCACIÓN SUPERIOR, CONPAB-IES (2005). Normas para bibliotecas de instituciones de educación superior e investigación. Guadalajara, México: CONPAB-IES.

FUSHMINI, MALLO, J., PICHININI, M. (2005). Memoria académica y cientifica: el rol de la biblioteca universitaria en la preservación y difusión generado en las universidades. Ponencia presentada en las Cuartas Jornadas de Sociología de la UNLP: La Argentina de la Crisis. La Plata, Argentina: Universidad Nacional de la Plata, Facultad de Humanidades y Ciencias de la Educación. 
GALAZ FONTES, J.F. (1998). Sobre la clasificación de las instituciones mexicanas de educación superior. Revista de la educación Superior, 106 (27).

GÓMEZ. J. (2012). Normas para bibliotecas universitarias españolas. Rebiun.

LARA ORDUÑO, S. (2004). La biblioteca de la Universidad Mexicana, plantel Satélite: un apoyo hacia el sistema de ingreso y permanencia en la FIMPES. Informe Académico de Licenciatura en Biblioteconomía. México, D. F.: Secretaría de Educación Escuela, Dirección General de Educación Superior, Nacional de Biblioteconomía y Archivonomía, Pública.

LAU, J. (2001). Aprendizaje y calidad educativa: el papel de las bibliotecas. Ponencia presentada en Seminario de Bibliotecas y Calidad de la Educación, Universidad de Medellín, Colombia, mayo, 2. http://bivir.uacj.mx/dhi/ PublicacionesUACJ/Docs/Ponencias/PDF/ponmedellin01.pdf [Consulta: 25/05/2013]

LOPERA, G. (2008). Reflexiones sobre el papel de la biblioteca en la calidad de la educación. Ponencia presentada en el Seminario "Estrategias de la Enseñanza Universitaria de la Universidad Nacional de Colombia. http://docencia.udea.edu.co/biblioteca/formacionusuarios/guiadelcurso/bibliografiadocumentos/Refelxiones\%20entorno....pdf [Consulta: 25/05/2013]

LÓPEZ RUELAS, S., CORTÉS, J. (2011). Las normas para bibliotecas universitarias de CONPAB-IES: su estructura e intenciones. En: Lau, J., comp. Satisfacción de Usuarios: Evaluación Integral de Bibliotecas. (pp. 293-306). Xalapa, Ver: Universidad Veracruzana; México, D. F.: Library Outsourcing; Buenos Aires: Alfagrama Ediciones.

PASADAS UREÑA, C. (2004, junio). Normas para bibliotecas de instituciones de educación superior: aprobadas por la Junta de Gobierno de la ACRL-ALA. Boletín de la Asociación Andaluza de Bibliotecarios, 19 (77): 61-78.

ROE, E. (1981). Academics, librarians, resource management and resource use. West Yorkshire, England: British Library Lending Division.

SECRETARÍA DE EDUCACIÓN PÚBLICA. Educación superior pública, universidades públicas mexicanas. http://www.ses.sep.gob.mx/wb/ses/universidades_publicas_estatales [Consulta: 25/05/2013]

TARANGO, J. (2010). Diseño de un modelo de evaluación de desarrollo bibliotecario y propuesta para su implantación en el sistema universitario UACH. (Tesis, Doctorado en Educación, Universidad Autónoma de Chihuahua, 2010).

TARANGO, J. y HERNÁNDEZ-OROZCO, G. (2009). Evaluación de bibliotecas universitarias: un modelo de avance y desarrollo. Revista Interamericana de Bibliotecología, 32 (2): 147-175.

UNIVERSIDAD NACIONAL AUTÓNOMA DE MÉXICO. DIRECCIÓN GENERAL DE EVALUACIÓN INSTITUCIONAL. (2012). Estudio comparativo de las universidades mexicanas. http://www.execum.unam.mx/ [Consulta: 25/05/2013] 
WEBB, S. P., GRIMWOOD-JONES, D. (2003). Personal development in the information and library profession. Londres, Inglaterra: Europe Publications.

WILBURN, N. (2004). Human Resource management in today's academic library. Nueva York: Libraries Unlimited.

Anexo 1. Lista de universidades participantes

\begin{tabular}{|c|c|c|}
\hline UNIVERSIDAD & $\begin{array}{c}\text { RÉGIMEN } \\
\text { FINANCIERO }\end{array}$ & $\begin{array}{c}\text { TIPO DE } \\
\text { INSTITUCIÓN }\end{array}$ \\
\hline 1. Benemérita Universidad Autónoma de Puebla & Pública Estatal & Grande \\
\hline 2. El Colegio de México & Pública Federal & Pequeña \\
\hline 3. El Colegio de Michoacán & Pública Federal & Pequeña \\
\hline 4. El Colegio de Sonora & Publica Estatal & Pequeña \\
\hline 5. Instituto Tecnológico de Sonora & Pública Federal & Mediana \\
\hline 6. Universidad Autónoma de Aguascalientes & Pública Estatal & Pequeña \\
\hline 7. Universidad Autónoma de Baja California Sur & Pública Estatal & Pequeña \\
\hline 8. Universidad Autónoma de Campeche & Pública Estatal & Pequeña \\
\hline 9. Universidad Autónoma de Chiapas & Pública Estatal & Mediana \\
\hline 10. Universidad Autónoma de Chihuahua & Pública Estatal & Mediana \\
\hline 11. Universidad Autónoma de Ciudad Juárez & Pública Estatal & Mediana \\
\hline 12. Universidad Autónoma de Nayarit & Pública Estatal & Pequeña \\
\hline 13. Universidad Autónoma de Nuevo León & Pública Estatal & Grande \\
\hline 14. Universidad Autónoma de Querétaro & Pública Estatal & Mediana \\
\hline 15. Universidad Autónoma de San Luis Potosí & Pública Estatal & Mediana \\
\hline 16. Universidad Autónoma de Sinaloa & Pública Estatal & Grande \\
\hline 17. Universidad Autónoma de Tamaulipas & Pública Estatal & Mediana \\
\hline 18. Universidad Autónoma de Tlaxcala & Pública Estatal & Pequeña \\
\hline 19. Universidad Autónoma de Yucatán & Pública Estatal & Pequeña \\
\hline 20. Universidad Autónoma de Zacatecas & Pública Estatal & Mediana \\
\hline 21. Universidad Autónoma del Carmen & Pública Estatal & Pequeña \\
\hline 22. Universidad Autónoma del Estado de Hidalgo & Pública Estatal & Mediana \\
\hline 23. Universidad Autónoma del Estado de México & Pública Estatal & Grande \\
\hline 24. Universidad de Ciencias y Artes de Chiapas & Pública Estatal & Pequeña \\
\hline 25. Universidad de Colima & Pública Estatal & Pequeña \\
\hline 26. Universidad de Guadalajara & Pública Estatal & Grande \\
\hline 27. Universidad de Guanajuato & Pública Estatal & Mediana \\
\hline 28. Universidad de Monterrey & Privada & Pequeña \\
\hline 29. Universidad de Quintana Roo & Pública Estatal & Pequeña \\
\hline 30. Universidad de Sonora & Pública Estatal & Mediana \\
\hline 31. Universidad Juárez Autónoma de Tabasco & Pública Estatal & Mediana \\
\hline 32. Universidad Michoacana de S.Nicolás de Hidalgo & Pública Estatal & Mediana \\
\hline 33. Universidad Veracruzana & Pública Estatal & Grande \\
\hline
\end{tabular}


Anexo 2. Índices de correlación de variables

\begin{tabular}{|c|c|c|c|c|c|c|}
\hline \multirow{2}{*}{\multicolumn{2}{|c|}{$\begin{array}{c}\text { Índices de infraestructura } \\
\text { bibliotecaria }\end{array}$}} & \multicolumn{5}{|c|}{ Índices de producción científica*** } \\
\hline & & 1 & 2 & 3 & 4 & 5 \\
\hline \multirow{3}{*}{$\begin{array}{l}\text { Número de volúmenes } \\
\text { impresos por cada } \\
\text { profesor }\end{array}$} & $\begin{array}{l}\text { Correlación de } \\
\text { Pearson }\end{array}$ & $.686^{* *}$ & $.934^{* *}$ & -.092 & $.845^{* *}$ & $.845^{* *}$ \\
\hline & Sig. (bilateral) & .000 & .000 & .612 & .000 & .000 \\
\hline & $\mathrm{N}$ & 33 & 33 & 33 & 33 & 33 \\
\hline \multirow{3}{*}{$\begin{array}{l}\text { Número de } \\
\text { suscripciones a revistas } \\
\text { impresas por cada } 100 \\
\text { profesores }\end{array}$} & $\begin{array}{l}\text { Correlación de } \\
\text { Pearson }\end{array}$ & $.346^{*}$ & $.532^{* *}$ & -.127 & $.454^{* *}$ & $.454^{* *}$ \\
\hline & Sig. (bilateral) & .049 & .001 & .480 & .008 & .008 \\
\hline & $\mathrm{N}$ & 33 & 33 & 33 & 33 & 33 \\
\hline \multirow{3}{*}{$\begin{array}{l}\text { Número de } \\
\text { suscripciones a bases } \\
\text { de datos por cada } 100 \\
\text { profesores }\end{array}$} & $\begin{array}{l}\text { Correlación de } \\
\text { Pearson }\end{array}$ & $.584^{* *}$ & $.762^{* *}$ & -.134 & $.690^{* *}$ & $.690^{* *}$ \\
\hline & Sig. (bilateral) & .000 & .000 & .459 & .000 & .000 \\
\hline & $\mathrm{N}$ & 33 & 33 & 33 & 33 & 33 \\
\hline \multirow{3}{*}{$\begin{array}{l}\text { Número de usuarios } \\
\text { docentes por cada } 100 \\
\text { profesores }\end{array}$} & $\begin{array}{l}\text { Correlación de } \\
\text { Pearson }\end{array}$ & -.074 & -.098 & -.012 & -.093 & -.093 \\
\hline & Sig. (bilateral) & .684 & .587 & .947 & .608 & .608 \\
\hline & $\mathrm{N}$ & 33 & 33 & 33 & 33 & 33 \\
\hline \multirow{3}{*}{$\begin{array}{l}\text { Número de usuarios } \\
\text { estudiantes por cada } \\
100 \text { profesores }\end{array}$} & $\begin{array}{l}\text { Correlación de } \\
\text { Pearson }\end{array}$ & -.170 & -.259 & -.030 & -.235 & -.235 \\
\hline & Sig. (bilateral) & .343 & .146 & .868 & .187 & .187 \\
\hline & $\mathrm{N}$ & 33 & 33 & 33 & 33 & 33 \\
\hline \multirow{5}{*}{$\begin{array}{l}\text { Número de metros } \\
\text { cuadrados de } \\
\text { infraestructura física } \\
\text { bibliotecaria por cada } \\
100 \text { profesores }\end{array}$} & $\begin{array}{l}\text { Correlación de } \\
\text { Pearson }\end{array}$ & $.623^{* *}$ & $.749^{* *}$ & -.046 & $.708^{* *}$ & $.708^{* *}$ \\
\hline & Sig. (bilateral) & .000 & .000 & .798 & .000 & .000 \\
\hline & $\mathrm{N}$ & 33 & 33 & 33 & 33 & 33 \\
\hline & Sig. (bilateral) & .000 & .000 & .135 & .000 & \\
\hline & $\mathrm{N}$ & 33 & 33 & 33 & 33 & 33 \\
\hline
\end{tabular}

Notas:

* La correlación es significativa al nivel de 0,05 (bilateral)

** La correlación es significativa al nivel de 0,01 (bilateral)

*** Índices de producción científica:

$1=$ Índice de Productividad (Alumnos + Artículos + Revistas)

2 = Índice de Calidad (SNI+ CA PROMEP + Programas acreditados)

3 = Índice de Innovación (Patentes aprobadas + Patentes solicitadas)

4 = Índice de Competitividad $=$ Productividad + Calidad + Innovación

$5=$ Índice de Competitividad Ponderada $=0.33$ (Productividad $)+0.33($ Calidad $)+0.33($ Innovación $)$ 